\title{
Thermodynamics of Various Entropies in Specific Modified Gravity with Particle Creation
}

\author{
Abdul Jawad (D), Shamaila Rani (D), and Salman Rafique \\ Department of Mathematics, COMSATS University Islamabad, Lahore Campus, Lahore, Pakistan \\ Correspondence should be addressed to Shamaila Rani; drshamailarani@cuilahore.edu.pk
}

Received 27 February 2018; Revised 27 June 2018; Accepted 4 July 2018; Published 18 October 2018

Academic Editor: Chao-Qiang Geng

Copyright (c) 2018 Abdul Jawad et al. This is an open access article distributed under the Creative Commons Attribution License, which permits unrestricted use, distribution, and reproduction in any medium, provided the original work is properly cited. The publication of this article was funded by SCOAP S $^{3}$

\begin{abstract}
We consider the particle creation scenario in the dynamical Chern-Simons modified gravity in the presence of perfect fluid equation of state $p=(\gamma-1) \rho$. By assuming various modified entropies (Bekenstein entropy logarithmic entropy, power law correction, and Renyi entropy), we investigate the first law of thermodynamics and generalized second law of thermodynamics on the apparent horizon. In the presence of particle creation rate, we discuss the generalized second law of thermodynamics and thermal equilibrium condition. It is found that thermodynamics laws and equilibrium condition remain valid under certain conditions of parameters.
\end{abstract}

\section{Introduction}

Recently, different observations such as cosmic microwave background radiations (CMBR) [1] and sloan digital sky survey (SDSS) [2] have confirmed the accelerated expansion of universe. It is predicted that the expansion of the universe is due to the curious form of force, i.e., dark energy (DE). This discovery was unexpected, because before this invention cosmologists just think that the expansion of the universe would be decelerating because of the gravitational attraction of the matter in the universe. Scientists have proposed various aspects of dynamical DE models such as quintessence [3], K-essence [4], phantom [5], quintom [6], tachyon [7], holographic [8], and pilgrim DE [9-13]. The simplest candidate of $\mathrm{DE}$ is cosmological constant but its formation and mechanisms are unknown. In order to explain the cosmic acceleration, various DE models and modified theories of gravity have been predicted such as $f(R), f(T)$ [14], $f\left(T, T_{G}\right)[15,16], f(T, T)[17,18]$, and dynamical ChernSimons modified gravity [19-22].

In cosmology, attempts to disclose the connection between Einstein gravity and thermodynamics were carried out in [23-32]. The basic concept of thermodynamics comes from black hole physics. In general, there has been some profound thought on the connection among gravity and thermodynamics for a long time. The initial work was done by
Jacobson who showed that the gravitational Einstein equation can be derived from the relation between the horizon area and entropy, together with the Clausius relation $\delta Q=T \delta S$ (where $\delta Q, T$, and $\delta S$ represent the change in energy, temperature, and entropy change o the system, respectively) [33]. Further, various gravity theories have been investigated to study the deep connection between gravity and thermodynamics [34-41]. Cosmological investigations of thermodynamics in modified gravity theories have been executed in [11, 4254] (for a recent review on thermodynamic properties of modified gravity theories, see, e.g., [55]). Saha and Mondal [56] have studied thermodynamics on apparent horizon with the help of gravitationally induced particle scenario with constant specific entropy and particle creation rate $\Gamma$.

In the present work, we develop the particle creation scenario in the dynamical Chern-Simons modified gravity by assuming various modified entropies (Bekenstein entropy logarithmic entropy, power law correction, and Renyi entropy) on the apparent horizon. In the presence of particle creation rate, we discuss the generalized second law of thermodynamics (GSLT). This paper is organized as follows: in the next section, we will present the basic equations of dynamical Chern-Simons modified theory and particle creation rate. Also, we investigate the first law of thermodynamics. Sections 3 and 4 contain the illustration of GSLT. In Section 5, we analyze the stability of thermodynamical 
equilibrium for constant as well as variable $\Gamma$. In the last section, we summarize our results.

\section{Modified Entropies and Particle Creation Rate}

It was shown that the differential form of the Friedmann equation in the FRW universe can be written in the form of the first law of thermodynamics on the apparent horizon. The profound connection provides a thermodynamical interpretation of gravity which makes it interesting to explore the cosmological properties through thermodynamics. It was proved that for any spherically symmetric spacetime the field equations can be expressed as $T d S=d E+P d V$ for any horizon [37], where $E, P$, and $V$ represent the internal energy, pressure, and volume of the spherical system, respectively. The generalized second law of thermodynamics (GSLT) has been studied extensively in the behavior of expanding universe. According to GSLT "the sum of all entropies of the constituents (mainly DM and DE) and entropy of boundary (either it is Hubble or apparent or event horizons) of the universe can never decrease" [57]. Most of the researchers have discussed the validity of GSLT of different system including interaction of two fluid components dark energy (DE) and dark matter (DM) [58-61] and interaction of three fluid components [62] in FRW universe.

To discuss the behavior of GSLT scientist assumed horizon entropy $1 / 4$ of its area [63], power law correction $[64,65]$, logarithmic entropy [66], and Renyi entropy. GSLT has been discussed on the basis of gravitationally induced particle scenario which was firstly introduced by Schrodinger [67] on microscopic level. Parker et al. [68-72] extend this mechanism towards quantum field theory in curved space. Prigogine et al. [73] introduced the macroscopic mechanism of gravitationally induced particle scenario. Afterward, covariant description and difference between particle creation and bulk viscosity of creation process were given [74]. The particle creation process can be predicted with the incorporation of backreaction term in the Einstein field equations whose negative weight may help in clarifying the cosmic acceleration. In such a way, most of the phenomenological models of particle creation have been granted $[75,76]$. In addition, it was proved that phenomenological particle creation helps us to discuss the behavior of accelerating universe and paved the alternative way to the concordance $\Lambda$ CDM model [77, 78].

In the following discussion we will analyze the validity of first law of thermodynamics with Gibbs relation, GSLT, and thermodynamical equilibrium by assuming the following entropy corrections.

(i) Bekenstein entropy: the Bekenstein entropy and Hawking temperature of the apparent horizon are given by $(8 \pi=G=1)$

$$
\begin{aligned}
& S_{A}=\frac{A}{4}=\frac{R_{A}^{2}}{8}, \\
& T_{A}=\frac{1}{2 \pi R_{A}}=\frac{4}{R_{A}}
\end{aligned}
$$

where $A=4 \pi R_{A}^{2}$.

(ii) Logarithmic corrected entropy: to study the expansion of entropy of the universe, we discuss the addition of entropy related to the horizon. Quantum gravity allows the logarithmic corrections in the presence of thermal equilibrium fluctuations and quantum fluctuations [79-85]. The logarithmic entropy corrections can be defined as

$$
S_{A}=\frac{A}{4 L_{p}^{2}}+\alpha \ln \frac{A}{4 L_{p}^{2}}+\beta \frac{4 L_{p}^{2}}{A},
$$

where $L_{P}$ is the plank's length and $\alpha, \beta$ are constants whose values are still under consideration.

(iii) Power law correction: in thermodynamics of apparent horizon in the standard FRW cosmology, the geometric entropy is assumed to be proportional to its horizon area $\left(S_{A}=A / 4\right)$. The quantum corrections provided to the entropy-area relationship lead to the curvature correction in the Einstein-Hilbert action and vice versa $[83,86,87]$. The power law quantum correction to the horizon entropy motivated by the entanglement of quantum fields between inside and outside of the horizon is given by [88]

$$
S_{A}=\frac{A}{4 L_{p}^{2}}\left(1-K_{\delta} A^{1-\delta / 2}\right),
$$

where $K_{\delta}=\delta(4 \pi)^{\delta / 2-1} /(4-\delta) r_{c}^{4-\delta}, \delta$ is dimensionless constant, and $r_{c}$ is the crossover scale.

(iv) Renyi entropy: a novel sort of Renyi entropy has been proposed and inspected [89-91], in which not exclusively is the logarithmic corrected entropy of the original Renyi entropy utilized, yet the Bekenstein Hawking entropy $S_{B H}$ is thought to be a nonextensive Tsallis entropy $S_{A}$. One can obtain Renyi entropy $S_{R}$ [91]

$$
S_{R}=\frac{\ln \left(1+\eta S_{A}\right)}{\eta}
$$

The action of Chern-Simons theory is given by [19-21]

$$
\begin{aligned}
S= & \frac{1}{16 \pi G} \int d^{4} x\left[\sqrt{-g} R+\frac{\ell}{4} \theta^{\star} R^{\rho \sigma \mu \nu} R_{\rho \sigma \mu \nu}\right. \\
& \left.-\frac{1}{2} g^{\mu \nu} \nabla_{\mu} \theta \nabla \nu \theta+V(\theta)\right]+S_{m a t},
\end{aligned}
$$

where $R,{ }^{\star} R^{\rho \sigma \mu \nu} R_{\rho \sigma \mu \nu}, \ell, \theta, S_{\text {mat }}$, and $V(\theta)$ are Ricci scalar, a topological invariant called the Pontryagin term, the coupling constant, the dynamical variable, the action of matter, and the potential, respectively. The Friedmann equation for flat universe turns out to be [22]

$$
H^{2}=\frac{1}{3} \rho+\frac{c^{2}}{6 a^{6}},
$$


where $c$ is constant, $H=\dot{a}(t) / a(t)$ is a Hubble parameter, and $a(t)$ is the scale factor. The equation of continuity for this model can be described as

$$
\dot{\rho}+\Theta(\rho+P+\Pi)=0 .
$$

The particle creation pressure $(\Pi)$ representing the gravitationally induced process for particle creation and $\Theta=3 \mathrm{H}$ is the fluid expansion. The total number of $n$-particles in an open thermodynamics is

$$
\dot{n}+\Theta n=n \Gamma,
$$

where $\Gamma$ is the creation rate of number of particles in comoving volume, i.e., $\left(N=n a^{3}\right)$ having two phases negative and positive. The negative $\Gamma$ relates with the particle annihilation and the positive $\Gamma$ relates to production of particle. Equations (7) and (8) with Gibbs relations can be written as

$$
T d s=d\left(\frac{\rho}{n}\right)+p d\left(\frac{1}{n}\right) .
$$

The equation related to creation pressure $\Pi$ and $\Gamma$ can be determined as

$$
\Pi=-\frac{\Gamma}{\Theta}(\rho+p) .
$$

Under traditional assumption, the specific entropy of each particle is constant, i.e., the process is adiabatic or isentropic, which implies that dissipative fluid is similar to a perfect fluid with a nonconserved particle number. The respective EoS for this model is represented by $p=(\gamma-1) \rho$. Differentiation of (6) will give

$$
\dot{H}=-\frac{1}{2}\left((\rho+p+\Pi)-H \frac{c^{2}}{a^{6}}\right) .
$$

Inserting (10) and $p=(\gamma-1) \rho$ in the above equation, we get

$$
\frac{\dot{H}}{H^{2}}=-\frac{1}{2 H^{2}}\left(\gamma\left(3 H^{2}-\frac{c^{2}}{2 a^{6}}\right)\left(1-\frac{\Gamma}{3 H}\right)+\frac{c^{2}}{a^{6}}\right) .
$$

For flat FRW universe, Hubble parameter relates to the apparent horizon as $R_{A}=1 / H$. Differentiating the apparent horizon with respect to time, we have

$$
\begin{aligned}
\dot{R}_{A} & =-\frac{\dot{H}}{H^{2}} \\
& =\frac{1}{2 H^{2}}\left(\gamma\left(3 H^{2}-\frac{c^{2}}{2 a^{6}}\right)\left(1-\frac{\Gamma}{3 H}\right)+\frac{c^{2}}{a^{6}}\right) .
\end{aligned}
$$

The deceleration parameter $q$ is of the form

$$
\begin{aligned}
q & =-\frac{\dot{H}}{H^{2}}-1 \\
& =\frac{1}{2 H^{2}}\left(\gamma\left(3 H^{2}-\frac{c^{2}}{2 a^{6}}\right)\left(1-\frac{\Gamma}{3 H}\right)+\frac{c^{2}}{a^{6}}\right)-1 .
\end{aligned}
$$

Next, we investigate the first law of thermodynamics in the presence of modified entropies. The relation between thermodynamics and Einstein field equations was found by Jacobson with the help of Clausius relation at apparent horizon described as

$$
-d E_{\mathrm{A}}=T_{\mathrm{A}} d S_{\mathrm{A}}
$$

For the sake of convenience, we consider $X=T_{\mathrm{A}} d S_{\mathrm{A}}+d E_{\mathrm{A}}$. The differential $d E_{\mathrm{A}}$ is the amount of energy crossing the apparent horizon which can be evaluated as [28]

$$
-d E_{\mathrm{A}}=\frac{1}{2} R^{3}(\rho+p) H d t=\frac{\gamma}{2 H^{2}}\left(3 H^{2}-\frac{c^{2}}{2 a^{6}}\right) .
$$

Bekenstein Entropy. From (1), the differential of surface entropy at apparent horizon leads to

$$
d S_{A}=\frac{1}{8 H^{3}}\left(\gamma\left(3 H^{2}-\frac{c^{2}}{2 a^{6}}\right)\left(1-\frac{\Gamma}{3 H}\right)+\frac{c^{2}}{a^{6}}\right) .
$$

The above equation with horizon temperature leads to

$$
T_{A} d S_{A}=\frac{1}{2 H^{2}}\left(\gamma\left(3 H^{2}-\frac{c^{2}}{2 a^{6}}\right)\left(1-\frac{\Gamma}{3 H}\right)+\frac{c^{2}}{a^{6}}\right) .
$$

Hence $X$ becomes

$$
\begin{aligned}
X= & -\frac{\gamma}{2 H^{2}}\left(3 H^{2}-\frac{c^{2}}{2 a^{6}}\right) \\
& +\frac{1}{2 H^{2}}\left(\gamma\left(3 H^{2}-\frac{c^{2}}{2 a^{6}}\right)\left(1-\frac{\Gamma}{3 H}\right)+\frac{c^{2}}{a^{6}}\right) .
\end{aligned}
$$

With the help of (19) we observe that the first law of thermodynamics holds (i.e., $X \longrightarrow 0)$ when $\Gamma=3 H\left(\left(c^{2} / \gamma a^{6}\right)\left(3 H^{2}-\right.\right.$ $\left.\left.c^{2} / 2 a^{6}\right)^{-1}\right)$.

Logarithmic Corrected Entropy. The differential form of (2) is given as

$$
d S_{A}=\frac{\left(\gamma\left(3 H^{2}-c^{2} / 2 a^{6}\right)(1-\Gamma / 3 H)+c^{2} / a^{6}\right)\left(1 / 4 H L_{\mathrm{p}}^{2}+2 \alpha H-16 \beta H^{3} L_{\mathrm{p}}^{2}\right)}{2 H^{2}} d t
$$


which leads to

$$
\begin{aligned}
T_{A} d S_{A}= & \frac{1}{2 H^{2}}\left(\gamma\left(3 H^{2}-\frac{c^{2}}{2 a^{6}}\right)\left(1-\frac{\Gamma}{3 H}\right)+\frac{c^{2}}{a^{6}}\right) \\
& \cdot\left(\frac{1}{L_{\mathrm{p}}^{2}}+8 H^{2} \alpha-64 \beta H^{4} L_{\mathrm{p}}^{2}\right) .
\end{aligned}
$$

Combining (16) and (21), we get

$$
\begin{aligned}
X= & \frac{1}{2 H^{2}}\left(\gamma\left(3 H^{2}-\frac{c^{2}}{2 a^{6}}\right)\left(1-\frac{\Gamma}{3 H}\right)+\frac{c^{2}}{a^{6}}\right) \\
& \cdot\left(\frac{1}{L_{\mathrm{p}}^{2}}+8 H^{2} \alpha-64 \beta H^{4} L_{\mathrm{p}}^{2}\right) \\
& -\frac{\gamma}{2 H^{2}}\left(3 H^{2}-\frac{c^{2}}{2 a^{6}}\right) .
\end{aligned}
$$

From the above equation, we observe the validity of first law of thermodynamics for $\Gamma=3 H\left(1-(1 / \gamma)\left(3 H^{2}-\right.\right.$ $\left.\left.c^{2} / a^{6}\right)^{-1}\left(\gamma\left(3 H^{2}-c^{2} / 2 a^{6}\right) /\left(1 / L_{\mathrm{p}}^{2}+8 H^{2} \alpha-64 \beta H^{4} L_{\mathrm{p}}^{2}\right)-c^{2} / a^{6}\right)\right)$.

Power Law Correction. Differentiating (3), we get

$$
\begin{aligned}
d S_{A}= & \frac{1}{2 H^{2}}\left(\gamma\left(3 H^{2}-\frac{c^{2}}{2 a^{6}}\right)\left(1-\frac{\Gamma}{3 H}\right)+\frac{c^{2}}{a^{6}}\right) \\
& \cdot\left(\frac{1}{4 H L_{\mathrm{p}}^{2}}-\frac{K_{\delta}}{4 L_{\mathrm{p}}^{2}}\left(2-\frac{\delta}{2}\right)\left(\frac{1}{H}\right)^{3-\delta}\right) d t,
\end{aligned}
$$

which yields

$$
\begin{gathered}
T_{A} d S_{A}=\frac{1}{2 H^{2}}\left(\gamma\left(3 H^{2}-\frac{c^{2}}{2 a^{6}}\right)\left(1-\frac{\Gamma}{3 H}\right)+\frac{c^{2}}{a^{6}}\right) \\
\cdot\left(\frac{1}{L_{\mathrm{p}}^{2}}-\left(2-\frac{\delta}{2}\right) \frac{K_{\delta}}{L_{p}^{2}}\left(\frac{1}{H}\right)^{2-\delta}\right) .
\end{gathered}
$$

Hence, $X$ takes the form

$$
\begin{aligned}
X= & \frac{1}{2 H^{2}}\left(\gamma\left(3 H^{2}-\frac{c^{2}}{2 a^{6}}\right)\left(1-\frac{\Gamma}{3 H}\right)+\frac{c^{2}}{a^{6}}\right) \\
& \cdot\left(\frac{1}{L_{\mathrm{p}}^{2}}-\left(2-\frac{\delta}{2}\right) \frac{K_{\delta}}{L_{p}^{2}}\left(\frac{1}{H}\right)^{2-\delta}\right) \\
& -\frac{\gamma}{2 H^{2}}\left(3 H^{2}-\frac{c^{2}}{2 a^{6}}\right) .
\end{aligned}
$$

From (25), it can be analyzed that the first law of thermodynamics remains valid for the following particle creation rate:

$$
\begin{aligned}
\Gamma= & 3 H\left(1-\frac{1}{\gamma}\left(3 H^{2}-\frac{c^{2}}{2 a^{6}}\right)^{-1}\left(\gamma\left(3 H^{2}-\frac{c^{2}}{2 a^{6}}\right)\right.\right. \\
& \left.\left.\cdot\left(\frac{1}{L_{\mathrm{p}}^{2}}-\left(2-\frac{\delta}{2}\right) \frac{K_{\delta}}{L_{p}^{2}}\left(\frac{1}{H}\right)^{2-\delta}\right)^{-1}-\frac{c^{2}}{a^{6}}\right)\right) .
\end{aligned}
$$

Renyi Entropy. Equation (4) gives the differential of entropy as

$$
\begin{aligned}
d S_{R}= & \frac{1}{H}\left(\gamma\left(3 H^{2}-\frac{c^{2}}{2 a^{6}}\right)\left(1-\frac{\Gamma}{3 H}\right)+\frac{c^{2}}{a^{6}}\right) \\
& \cdot\left(\frac{1}{\eta+8 H^{2}}\right),
\end{aligned}
$$

which gives rise to

$$
\begin{aligned}
& T_{A} d S_{R} \\
& \quad=\frac{4}{\left(\eta+8 H^{2}\right)}\left(\gamma\left(3 H^{2}-\frac{c^{2}}{2 a^{6}}\right)\left(1-\frac{\Gamma}{3 H}\right)+\frac{c^{2}}{a^{6}}\right) .
\end{aligned}
$$

Using (16) and (28), we get

$$
\begin{aligned}
X= & \frac{4}{\left(\eta+8 H^{2}\right)}\left(\gamma\left(3 H^{2}-\frac{c^{2}}{2 a^{6}}\right)\left(1-\frac{\Gamma}{3 H}\right)+\frac{c^{2}}{a^{6}}\right) \\
& -\frac{\gamma}{2 H^{2}}\left(3 H^{2}-\frac{c^{2}}{2 a^{6}}\right) .
\end{aligned}
$$

From (29), it can be seen that the first law of thermodynamics is showing the validity when $\Gamma=3 H\left(1-(1 / \gamma)\left(3 H^{2}-\right.\right.$ $\left.\left.c^{2} / 2 a^{6}\right)^{-1}\left(\left(\gamma\left(\eta+8 H^{2}\right) / 8 H^{2}\right)\left(3 H^{2}-c^{2} / 2 a^{6}\right)-c^{2} / a^{6}\right)\right)$.

\section{Generalized Second Law of Thermodynamics}

We discuss the GSLT of an isolated macroscopic physical system where the total entropy $S_{T}$ must satisfy the following conditions $d\left(S_{\mathrm{A}}+S_{\mathrm{f}}\right) \geq 0$; i.e., entropy function cannot be decreased. In this relation, $S_{\mathrm{A}}$ and $S_{\mathrm{f}}$ appear as the entropy at apparent horizon and the entropy of cosmic fluid enclosed within the horizon, respectively. The Gibbs equation is of the form

$$
T_{\mathrm{f}} d S_{\mathrm{f}}=d E_{\mathrm{f}}+p d V
$$

where $T_{\mathrm{f}}$ is the temperature of the cosmic fluid and $E_{\mathrm{f}}$ is the energy of the fluid $\left(E_{\mathrm{f}}=\rho V\right)$. The evolution equation for fluid temperature having constant entropy can be described as [92]

$$
\frac{\dot{T}_{\mathrm{f}}}{T_{\mathrm{f}}}=(\Gamma-\Theta) \frac{\partial p}{\partial \rho} .
$$

Using (12), we get $(\Gamma-\Theta)=\left(6 H \dot{H}+3 H c^{2} / a^{6}\right) / \gamma\left(3 H^{2}-\right.$ $\left.c^{2} / 2 a^{6}\right)$; hence, (31) leads to the integral

$$
\ln \left(\frac{T_{\mathrm{f}}}{T_{0}}\right)=\frac{\gamma-1}{\gamma} \int \frac{\left(2 H \dot{H}+H c^{2} / a^{6}\right)}{\left(H^{2}-c^{2} / 6 a^{6}\right)} d H .
$$

Integration of the above equation leads to

$$
T_{\mathrm{f}}=T_{0}\left(H^{2}-\frac{c^{2}}{6 a^{6}}\right)^{(\gamma-1) / \gamma}
$$




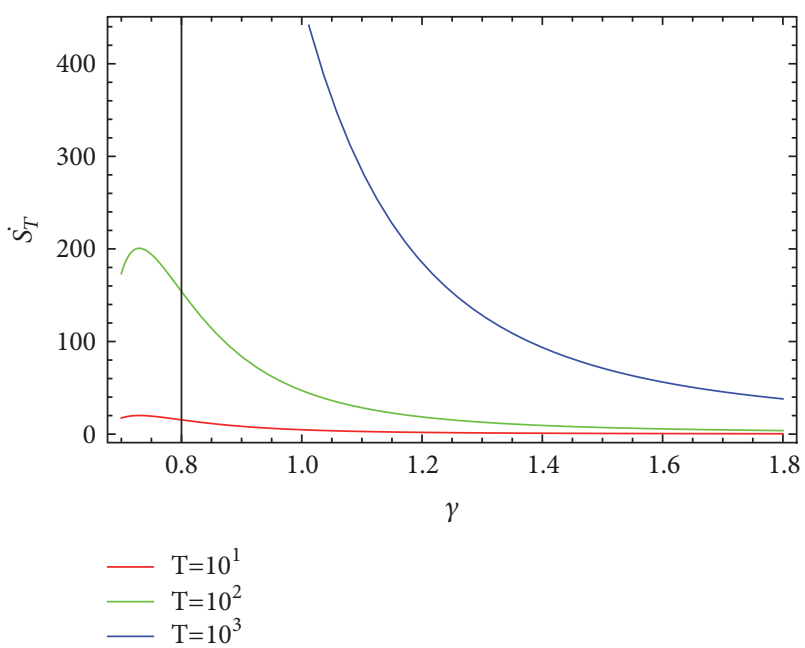

FIgURE 1: Plot of $\dot{S_{T}}$ versus $\gamma$ for Bekenstein entropy.

where $T_{0}$ is the constant of integration. Equation (30) yields the differential of fluid entropy as

$$
\begin{aligned}
d S_{f}= & \frac{T_{0}^{-1}}{H^{2}}\left(H^{2}-\frac{c^{2}}{6 a^{6}}\right)^{(1-\gamma) / \gamma} \\
& \cdot\left(\left(2 \dot{H}+\frac{c^{2}}{a^{6}}\right)-\frac{\gamma \dot{H}}{H^{2}}\left(3 H^{2}-\frac{c^{2}}{2 a^{6}}\right)\right) .
\end{aligned}
$$

Next, we observe the validity of GSLT by assuming Bekenstein entropy, logarithmic corrected entropy, power law correction, and Renyi entropy.

Bekenstein Entropy. In present case, we get the differential of total entropy by using (17) and (34) as

$$
\begin{aligned}
\dot{S_{T}}= & \frac{1}{8 H^{3}}\left(\gamma\left(3 H^{2}-\frac{c^{2}}{2 a^{6}}\right)\left(1-\frac{\Gamma}{3 H}\right)+\frac{c^{2}}{a^{6}}\right) \\
& +\frac{T_{0}^{-1}}{H^{2}}\left(H^{2}-\frac{c^{2}}{6 a^{6}}\right)^{(1-\gamma) / \gamma} \\
& \times\left(\left(2 \dot{H}+\frac{c^{2}}{a^{6}}\right)-\frac{\gamma \dot{H}}{H^{2}}\left(3 H^{2}-\frac{c^{2}}{2 a^{6}}\right)\right),
\end{aligned}
$$

where $S_{T}=S_{\mathrm{A}}+S_{\mathrm{f}}$. The plot of $S_{T}$ versus $\gamma$ is shown in Figure 1 for three values of $T$. We observe the validity of GSLT at the present epoch by setting the constant values $H=H_{0}=$ $67, c=-1, a=a_{0}=1$, and $q=-0.53$. It can be analyzed from the figure that $S_{T} \geq 0$ for all values of $T$ leads to the validity of GSLT.

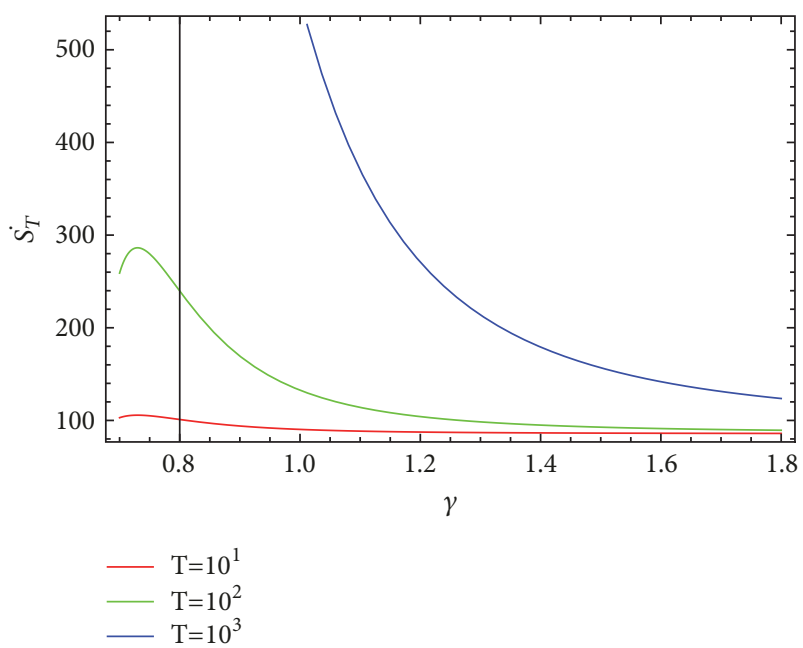

FIgURE 2: Plot of $\dot{S_{T}}$ versus $\gamma$ for logarithmic corrected entropy.

Logarithmic Corrected Entropy. Using (20) and (34), the differential of total entropy is given by

$$
\begin{aligned}
\dot{S_{T}}= & \frac{1}{2 H^{2}}\left(\gamma\left(3 H^{2}-\frac{c^{2}}{2 a^{6}}\right)\left(1-\frac{\Gamma}{3 H}\right)+\frac{c^{2}}{a^{6}}\right) \\
& \cdot\left(\frac{1}{4 H L_{p}^{2}}+2 \alpha H-16 \beta H^{3} L_{p}^{2}\right) d t \\
& +\frac{T_{0}^{-1}}{H^{2}}\left(H^{2}-\frac{c^{2}}{6 a^{6}}\right) \\
& \cdot\left(\left(2 \dot{H}+\frac{c^{2}}{a^{6}}\right)-\frac{\gamma \dot{H}}{H^{2}}\left(3 H^{2}-\frac{c^{2}}{2 a^{6}}\right)\right) d t .
\end{aligned}
$$

The plot of $\dot{S_{T}}$ versus $\gamma$ with respect to the three values of $T$ by fixing the constant values $\alpha=1, \beta=-0.00001$, and $L_{P}=1$ is shown in Figure 2. The trajectories in the plot remain which lead to the validity of GSLT in the presence of logarithmic entropy.

Power Law Correction. From (24) and (34), we get the differential of total entropy as

$$
\begin{aligned}
\dot{S_{T}}= & \frac{1}{2 H^{2}}\left(\gamma\left(3 H^{2}-\frac{c^{2}}{2 a^{6}}\right)\left(1-\frac{\Gamma}{3 H}\right)+\frac{c^{2}}{a^{6}}\right) \\
& \cdot\left(\frac{1}{4 H L_{\mathrm{p}}^{2}}-\frac{K_{\delta}}{4 L_{\mathrm{p}}^{2}}\left(2-\frac{\delta}{2}\right)\left(\frac{1}{H}\right)^{3-\delta}\right) d t \\
+ & \frac{T_{0}^{-1}}{H^{2}}\left(H^{2}-\frac{c^{2}}{6 a^{6}}\right)^{(1-\gamma) / \gamma} \\
& \cdot\left(\left(2 \dot{H}+\frac{c^{2}}{a^{6}}\right)-\frac{\gamma \dot{H}}{H^{2}}\left(3 H^{2}-\frac{c^{2}}{2 a^{6}}\right)\right) d t .
\end{aligned}
$$

The plot of $\dot{S_{T}}$ versus $\gamma$ for three values of $T$ by setting the constant values as $\delta=1, r_{c}=1 / 67$, and $L_{P}=1$ is shown 


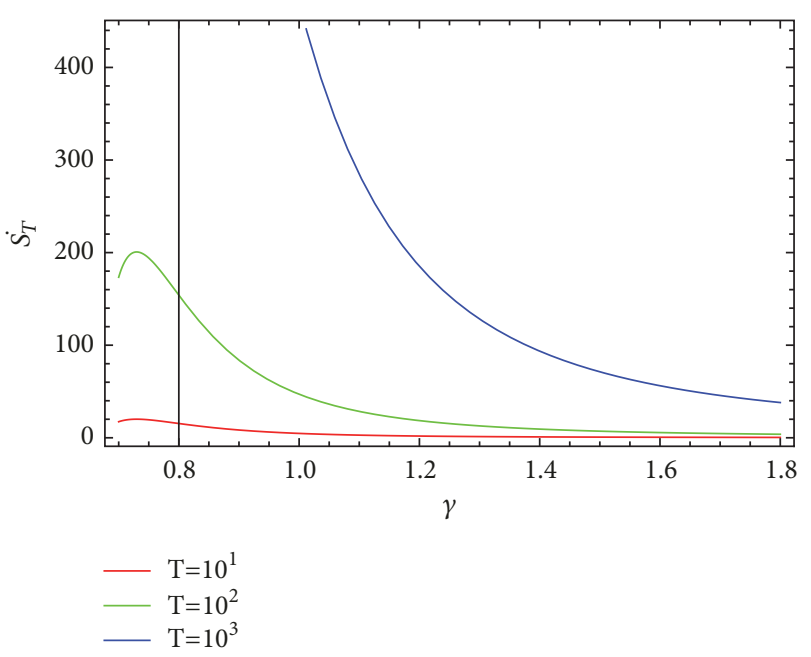

Figure 3: Plot of $\dot{S}_{T}$ versus $\gamma$ for power law correction.

in Figure 3. We can see $\dot{S}_{T}$ remains positive for all values of $T$ which leads to the validity of GSLT.

Renyi Entropy. We observe the validity of GSLT with $\dot{S_{T}} \geq 0$ for which (27) and (34) take the form

$\dot{S_{T}}$

$$
\begin{aligned}
= & \frac{1}{H\left(\eta+8 H^{2}\right)}\left(\gamma\left(3 H^{2}-\frac{c^{2}}{2 a^{6}}\right)\left(1-\frac{\Gamma}{3 H}\right)+\frac{c^{2}}{a^{6}}\right) \\
& +\frac{T_{0}^{-1}}{H^{2}}\left(H^{2}-\frac{c^{2}}{6 a^{6}}\right)^{(1-\gamma) / \gamma} \\
& \times\left(\left(2 \dot{H}+\frac{c^{2}}{a^{6}}\right)-\frac{\gamma \dot{H}}{H^{2}}\left(3 H^{2}-\frac{c^{2}}{2 a^{6}}\right)\right) .
\end{aligned}
$$

The plot of $S_{T}$ versus $\gamma$ for three values is shown in Figure 4 for $\eta=1$. It can be seen that GSLT is satisfying $\dot{S_{T}} \geq 0$ for all values of $T$ which leads to the validity of GSLT.

\section{Thermodynamical Equilibrium}

We discuss the two scenarios for thermodynamical equilibrium by taking particle creation rate $\Gamma=$ constant and $\Gamma=$ $\Gamma(t)$ for which entropy function attains a maximum entropy state, i.e., $d\left(S_{\mathrm{A}}+S_{\mathrm{f}}\right)<0$.

4.1. $\Gamma=$ Constant. Firstly, we consider $\Gamma$ as constant and observe the stability of thermodynamical equilibrium.

Bekenstein Entropy. We get second-order differential equation for constant $\Gamma$ by using (35):

$$
\begin{aligned}
\ddot{S_{T}} & =-\frac{3 \dot{H}\left(c^{2} / a^{6}+\gamma(1-\Gamma / 3 H)\left(-\left(c^{2} / 2 a^{6}\right)+3 H^{2}\right)\right)}{8 H^{4}}-2 \dot{H}\left(-\frac{c^{2}}{6 a^{6}}+H^{2}\right) \\
& \times \frac{\left(-\gamma(1-\Gamma / 3 H)\left(3 H^{2}-c^{2} / 2 a^{6}\right)+\gamma\left(3 H^{2}-c^{2} / 2 a^{6}\right)\left(c^{2} / a^{6}+\gamma(1-\Gamma / 3 H)\left(3 H^{2}-c^{2} / 2 a^{6}\right)\right) / 2 H^{2}\right)}{H^{3} T_{0}} \\
& +\frac{\left(-\gamma(1-\Gamma / 3 H)\left(3 H^{2}-c^{2} / 2 a^{6}\right)+\gamma\left(3 H^{2}-c^{2} / 2 a^{6}\right)\left(c^{2} / a^{6}+\gamma(1-\Gamma / 3 H)\left(3 H^{2}-c^{2} / 2 a^{6}\right)\right) / 2 H^{2}\right)}{\gamma H^{2} T_{0}} \times\left(\frac{c^{2} \dot{a}}{a^{7}}\right. \\
+ & 2 H \dot{H})(1-\gamma)\left(H^{2}-\frac{c^{2}}{6 a^{6}}\right)^{-1+(1-\gamma) / \gamma}+\gamma \Gamma \dot{H}\left(3 H^{2}-\frac{c^{2}}{2 a^{6}}\right) \frac{1}{24 H^{5}}-\frac{6 c^{2} \dot{a}}{8 H^{3} a^{7}}+\frac{\gamma(1-\Gamma / 3 H)\left(3 c^{2} \dot{a} / a^{7}+6 H \dot{H}\right)}{8 H^{3}} \\
& +\frac{\left(H^{2}-c^{2} / 6 a^{6}\right)(1-\gamma) / \gamma}{H^{2} T_{0}}\left(-\frac{\gamma \Gamma \dot{H}}{3 H^{2}} \times\left(3 H^{2}-\frac{c^{2}}{2 a^{6}}\right)-\frac{\gamma \dot{H}\left(3 H^{2}-c^{2} / 2 a^{6}\right)\left(c^{2} / a^{6}+\gamma(1-\Gamma / 3 H)\left(3 H^{2}-c^{2} / 2 a^{6}\right)\right)}{H^{3}}\right. \\
& \left.+\left(\frac{-6 c^{2} \dot{a} / a^{7}+\gamma \Gamma \dot{H}\left(3 H^{2}-c^{2} / 2 a^{6}\right) / 3 H^{2}}{2 H^{2}}+\frac{\left(3 c^{2} \dot{a} / a^{7}+6 H \dot{H}\right)}{2 H^{2}} \times \gamma\left(1-\frac{\Gamma}{3 H}\right)\right)\left(3 H^{2}-\frac{c^{2}}{2 a^{6}}\right) \gamma\right) .
\end{aligned}
$$

The graphical behavior of $\ddot{S_{T}}$ versus $\gamma$ is shown in Figure 5 for different values of parameter $T$. We observe that $S_{T}<0$ for all values of $T$ which leads to the validity of thermodynamical equilibrium. 


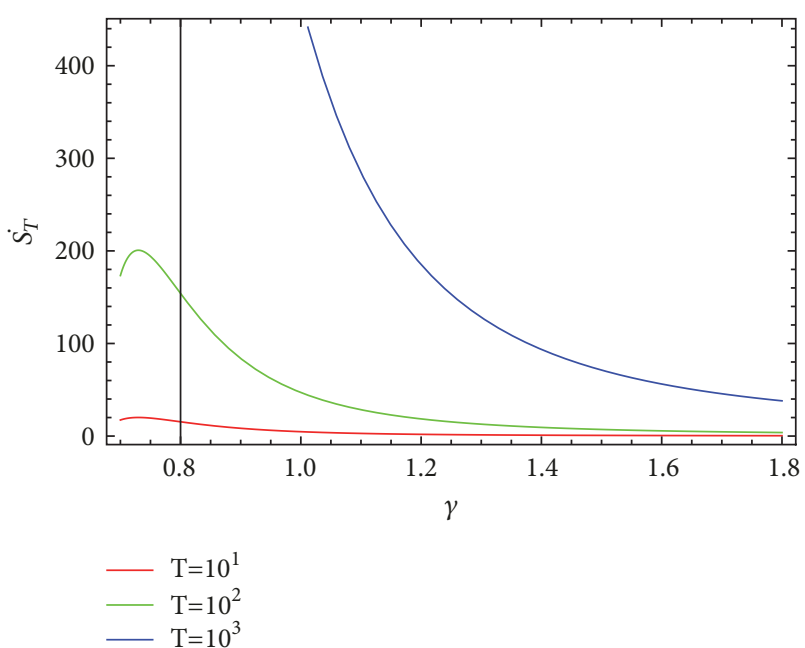

Figure 4: Plot of $\dot{S_{T}}$ versus $\gamma$ for Renyi entropy.

Logarithmic Corrected Entropy. In the presence of logarithmic corrected entropy second-order differential equation is obtained from (36) as

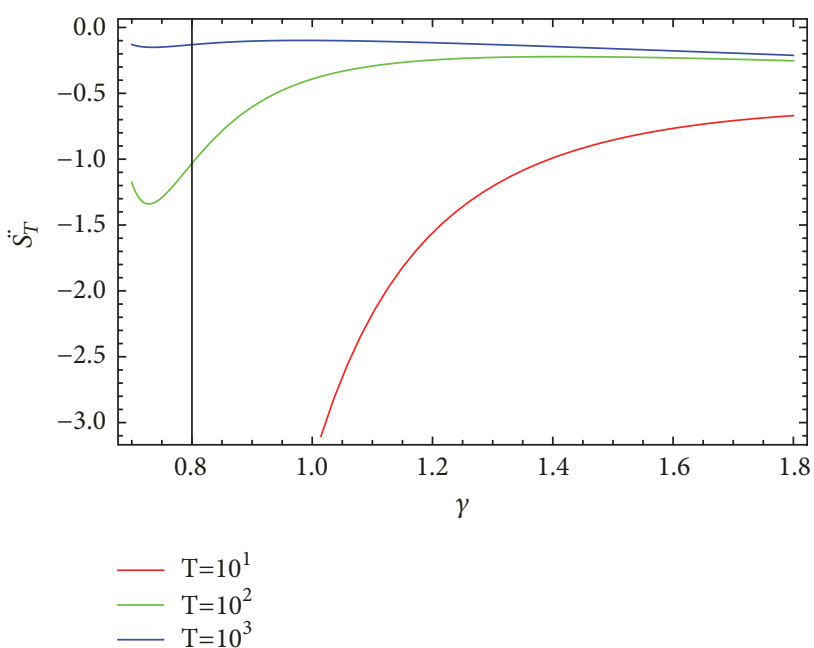

Figure 5: Plot of $\ddot{S_{T}}$ versus $\gamma$ for Bekenstein entropy when $\Gamma$ is constant.

$$
\begin{aligned}
\ddot{S_{T}} & =-\frac{\dot{H}\left(1 / 4 H L_{p}^{2}+2 \alpha H-16 L_{p}^{2} \beta H^{3}\right)\left(c^{2} / a^{6}+\gamma(1-\Gamma / 3 H)\left(3 H^{2}-c^{2} / 2 a^{6}\right)\right)}{H^{3}} \\
& -\left(\frac{-\gamma(1-\Gamma / 3 H)\left(3 H^{2}-c^{2} / 2 a^{6}\right)+\gamma\left(3 H^{2}-c^{2} / 2 a^{6}\right)\left(c^{2} / a^{6}+\gamma(1-\Gamma / 3 H)\left(3 H^{2}-c^{2} / 2 a^{6}\right)\right) / 2 H^{2}}{H^{3} T_{0}}\right) \times 2 \dot{H}\left(H^{2}\right. \\
& \left.-\frac{c^{2}}{6 a^{6}}\right)^{(1-\gamma) / \gamma}+(1-\gamma)\left(\frac{c^{2} \dot{a}}{a^{7}}+2 H \dot{H}\right)\left(H^{2}-\frac{c^{2}}{6 a^{6}}\right)^{-1+(1-\gamma) / \gamma} \\
& \times\left(\frac{-\gamma(1-\Gamma / 3 H)\left(3 H^{2}-c^{2} / 2 a^{6}\right)+\gamma\left(3 H^{2}-c^{2} / 2 a^{6}\right)\left(c^{2} / a^{6}+\gamma(1-\Gamma / 3 H)\left(3 H^{2}-c^{2} / 2 a^{6}\right)\right) / 2 H^{2}}{\gamma H^{2} T_{0}}\right) \\
& +\frac{\left(c^{2} / a^{6}+\gamma(1-\Gamma / 3 H)\left(3 H^{2}-c^{2} / 2 a^{6}\right)\right)\left(2 \alpha \dot{H}-\dot{H} / 4 L_{p}^{2} H^{2}-48 L_{p}^{2} \beta H^{2} \dot{H}\right)}{2 H^{2}}+\left(\frac{1}{4 H L_{p}^{2}}+2 \alpha H-16 L_{p}^{2} \beta H^{3}\right) \\
& \cdot\left(\frac{-6 c^{2} \dot{a} / a^{7}+\gamma(1-\Gamma / 3 H)\left(3 c^{2} \dot{a} / a^{7}+6 H \dot{H}\right)}{2 H^{2}}+\frac{\gamma \Gamma \dot{H}\left(3 H^{2}-c^{2} / 2 a^{6}\right)}{6 H^{4}}\right)+\frac{1}{H^{2} T_{0}}\left(H^{2}-\frac{c^{2}}{6 a^{6}}\right) \\
& \cdot\left(-\frac{\gamma \Gamma \dot{H}\left(3 H^{2}-c^{2} / 2 a^{6}\right)}{3 H^{2}}-\frac{\gamma \dot{H}\left(3 H^{2}-c^{2} / 2 a^{6}\right)\left(c^{2} / a^{6}+\gamma(1-\Gamma / 3 H)\left(3 H^{2}-c^{2} / 2 a^{6}\right)\right)}{H^{3}}-\gamma\left(1-\frac{\Gamma}{3 H}\right)\right. \\
& \left.+\gamma\left(3 H^{2}-\frac{c^{2}}{2 a^{6}}\right)\left(\frac{\gamma \Gamma \dot{H}\left(3 H^{2}-c^{2} / 2 a^{6}\right) / 3 H^{2}+\gamma(1-\Gamma / 3 H)\left(3 c^{2} \dot{a} / a^{7}+6 H \dot{H}\right)}{2 H^{2}}-\frac{6 c^{2} \dot{a}}{2 H^{2} a^{7}}\right)\right) . \\
& \times\left(\frac{3 c^{2} \dot{a}}{a^{7}}+6 H \dot{H}\right)+\frac{\gamma\left(3 c^{2} \dot{a} / a^{7}+6 H \dot{H}\right)\left(c^{2} / 2 a^{6}+\gamma(1-\Gamma / 3 H)\left(3 H^{2}-c^{2} / 2 a^{6}\right)\right)}{} \\
& +\left(\frac{H^{2}}{2}\right)
\end{aligned}
$$

The graphical behavior between $\ddot{S_{T}}$ and $\gamma$ is shown in Figure 6 for three values of $T$. We observe that all the trajectories are showing the negative increasing behavior, which exhibits the validity of thermodynamical equilibrium. 


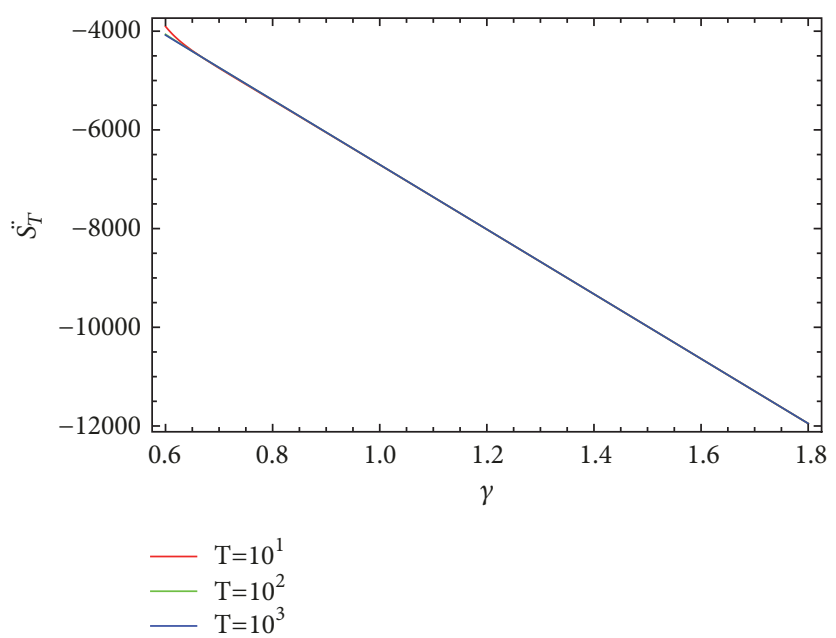

Figure 6: Plot of $\ddot{S_{T}}$ versus $\gamma$ for logarithmic corrected entropy when $\Gamma$ is a constant.

Power Law Correction. To find the validity of thermodynamical equilibrium, we take $\Gamma=$ constant for which second-order differential equation can be expressed with the help of (37) as

$$
\begin{aligned}
& \ddot{S_{T}}=-\frac{\left(c^{2} / a^{6}+\gamma(1-\Gamma / 3 H)\left(3 H^{2}-c^{2} / 2 a^{6}\right)\right)\left(1 / 4 H L_{p}^{2}-(2-\delta / 2)(1 / H)^{3-\delta} K_{\delta} / 4 L_{p}^{2}\right) \dot{H}}{H^{3}} \\
& -\left(\frac{-\gamma(1-\Gamma / 3 H)\left(3 H^{2}-c^{2} / 2 a^{6}\right)+\gamma\left(3 H^{2}-c^{2} / 2 a^{6}\right)\left(c^{2} / a^{6}+\gamma(1-\Gamma / 3 H)\left(3 H^{2}-c^{2} / 2 a^{6}\right)\right) / 2 H^{2}}{H^{3} T_{0}}\right) \times \dot{H}\left(H^{2}\right. \\
& \left.-\frac{c^{2}}{6 a^{6}}\right)^{(1-\gamma) / \gamma}+(1-\gamma)\left(\frac{c^{2} \dot{a}}{a^{7}}+2 H \dot{H}\right)\left(H^{2}-\frac{c^{2}}{6 a^{6}}\right)^{-1+(1-\gamma) / \gamma} \\
& \times\left(\frac{-\gamma(1-\Gamma / 3 H)\left(3 H^{2}-c^{2} / 2 a^{6}\right)+\gamma\left(3 H^{2}-c^{2} / 2 a^{6}\right)\left(c^{2} / a^{6}+\gamma(1-\Gamma / 3 H)\left(3 H^{2}-c^{2} / 2 a^{6}\right)\right) / 2 H^{2}}{\gamma H^{2} T_{0}}\right) \\
& +\frac{\left(c^{2} / a^{6}+\gamma(1-\Gamma / 3 H)\left(3 H^{2}-c^{2} / 2 a^{6}\right)\right)\left(-\dot{H} / 4 H L_{p}^{2}+(3-\delta) \dot{H}(2-\delta / 2)(1 / H)^{4-\delta} K_{\delta} / 4 L_{p}^{2}\right)}{2 H^{2}}+\left(\frac{1}{4 H L_{p}^{2}}\right. \\
& \left.-\frac{(2-\delta / 2)(1 / H)^{3-\delta} K_{\delta}}{4 H L_{p}^{2}}\right)\left(\frac{\left(-6 c^{2} \dot{a} / a^{7}\right)+\gamma(1-\Gamma / 3 H)\left(3 c^{2} \dot{a} / a^{7}+6 H \dot{H}\right)}{2 H^{2}}+\frac{\gamma \Gamma \dot{H}\left(3 H^{2}-c^{2} / 2 a^{6}\right)}{6 H^{4}}\right) \\
& +\frac{\left(H^{2}-c^{2} / 6 a^{6}\right)^{-(1-\gamma) / \gamma}}{H^{2} T_{0}}\left(\frac{\gamma \Gamma \dot{H}\left(3 H^{2}-c^{2} / 2 a^{6}\right)}{3 H^{2}}-\gamma\left(\frac{3 c^{2} \dot{a}}{a^{7}}+6 H \dot{H}\right)\left(1-\frac{\Gamma}{3 H}\right)\right. \\
& -\frac{\gamma \dot{H}\left(3 H^{2}-c^{2} / 2 a^{6}\right)\left(c^{2} / a^{6}+\gamma(1-\Gamma / 3 H)\left(3 H^{2}-c^{2} / 2 a^{6}\right)\right)}{H^{3}} \\
& +\frac{\gamma\left(3 c^{2} \dot{a} / a^{7}+6 H \dot{H}\right)\left(c^{2} / a^{6}+\gamma(1-\Gamma / 3 H)\left(3 H^{2}-c^{2} / 2 a^{6}\right)\right)}{2 H^{2}}+\gamma\left(3 H^{2}-\frac{c^{2}}{2 a^{6}}\right) \\
& \left.\times\left(\frac{-6 c^{2} \dot{a} / a^{7}+\gamma \Gamma \dot{H}\left(3 H^{2}-c^{2} / 2 a^{6}\right) / 3 H^{2}+\gamma(1-\Gamma / 3 H)\left(3 c^{2} \dot{a} / a^{7}+6 H \dot{H}\right)}{2 H^{2}}\right)\right) .
\end{aligned}
$$

The plot of $\ddot{S_{T}}$ versus $\gamma$ is shown in Figure 7 for three values of $T$. We analyze that thermodynamical equilibrium is satisfying the condition $\ddot{S}_{T}<0$ for all values of $T$, which leads to validity of thermodynamical equilibrium. 


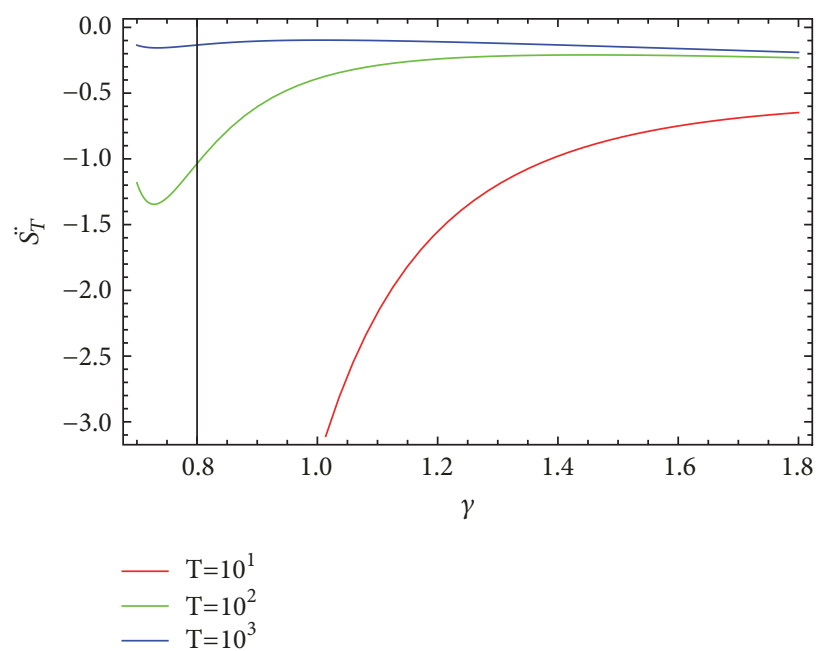

Figure 7: Plot of $\ddot{S_{T}}$ versus $\gamma$ for power law correction when $\Gamma$ is constant.

Renyi Entropy. In present scenario, we observe the validity of thermodynamical equilibrium by keeping $\Gamma$ as constant for which second-order differential equation is given by using (38) as

$$
\begin{aligned}
\dot{S}_{T}^{*} & =-\frac{16 \dot{H}}{\left(\eta+8 H^{2}\right)^{2}}\left(\frac{c^{2}}{a^{6}}+\gamma\left(3 H^{2}-\frac{c^{2}}{2 a^{6}}\right)\left(1-\frac{\Gamma}{3 H}\right)\right)-\frac{\dot{H}}{H^{2}\left(\eta+8 H^{2}\right)} \times\left(\frac{c^{2}}{a^{6}}+\gamma\left(3 H^{2}-\frac{c^{2}}{2 a^{6}}\right)\left(1-\frac{\Gamma}{3 H}\right)\right) \\
& -\frac{2 \dot{H}\left(H^{2}-c^{2} / 6 a^{6}\right)^{(1-\gamma) / \gamma}}{H^{3} T_{0}}\left(\left(3 H^{2}-\frac{c^{2}}{2 a^{6}}\right) \times\left(1-\frac{\Gamma}{3 H}\right)(-\gamma)\right. \\
& \left.+\frac{\gamma\left(3 H^{2}-c^{2} / 2 a^{6}\right)\left(c^{2} / a^{6}+\gamma\left(3 H^{2}-c^{2} / 2 a^{6}\right)(1-\Gamma / 3 H)\right)}{2 H^{2}}\right) \\
& +\left(\frac{\gamma\left(3 H^{2}-c^{2} / 2 a^{6}\right)\left(c^{2} / a^{6}+\gamma\left(3 H^{2}-c^{2} / 2 a^{6}\right)(1-\Gamma / 3 H)\right)}{2 H^{2}}-\gamma\left(3 H^{2}-\frac{c^{2}}{2 a^{6}}\right) \times\left(1-\frac{\Gamma}{3 H}\right)\right) \\
& \cdot \frac{(1-\gamma)\left(c^{2} \dot{a} / a^{7}+2 H \dot{H}\right)\left(H^{2}-c^{2} / 6 a^{6}\right)^{-1+(1-\gamma) / \gamma}}{\gamma H^{2} T_{0}}+\frac{1}{H\left(\eta+8 H^{2}\right)} \times\left(-\frac{6 c^{2} \dot{a}}{a^{7}}+\frac{\gamma \Gamma \dot{H}\left(3 H^{2}-c^{2} / 2 a^{6}\right)}{3 H^{2}}\right. \\
& \left.+\gamma\left(1-\frac{\Gamma}{3 H}\right)\left(\frac{3 c^{2} \dot{a}}{a^{7}}+6 H \dot{H}\right)\right)+\frac{1}{H^{2} T_{0}} \times\left(H^{2}-\frac{c^{2}}{6 a^{6}}\right)^{(1-\gamma) / \gamma} \\
& \cdot\left(-\frac{\gamma \dot{H}\left(3 H^{2}-c^{2} / 2 a^{6}\right)\left(c^{2} / a^{6}+\gamma\left(3 H^{2}-c^{2} / 2 a^{6}\right)(1-\Gamma / 3 H)\right)}{H^{3}}\right. \\
& +\frac{\gamma\left(3 c^{2} \dot{a} / a^{7}+6 H \dot{H}\right)\left(c^{2} / a^{6}+\gamma\left(3 H^{2}-c^{2} / 2 a^{6}\right)(1-\Gamma / 3 H)\right)}{2 H^{2}}-\frac{\gamma \Gamma \dot{H}\left(3 H^{2}-c^{2} / 2 a^{6}\right)}{3 H^{2}} \\
& +\gamma\left(1-\frac{\Gamma}{3 H}\right)\left(\frac{3 c^{2} \dot{a}}{a^{7}}+6 H \dot{H}\right) \\
& \left.\left.+\frac{\gamma c^{2} \dot{a}}{a^{7}}+\frac{\gamma \Gamma \dot{H}\left(3 H^{2}-c^{2} / 2 a^{6}\right)}{3 H^{2}}+\left(1-\frac{\Gamma}{3 H}\right) \times \gamma\left(\frac{3 c^{2} \dot{a}}{a^{7}}+6 H \dot{H}\right)\right) \frac{\gamma\left(3 H^{2}-c^{2} / 2 a^{6}\right)}{2 H^{2}}\right) .
\end{aligned}
$$




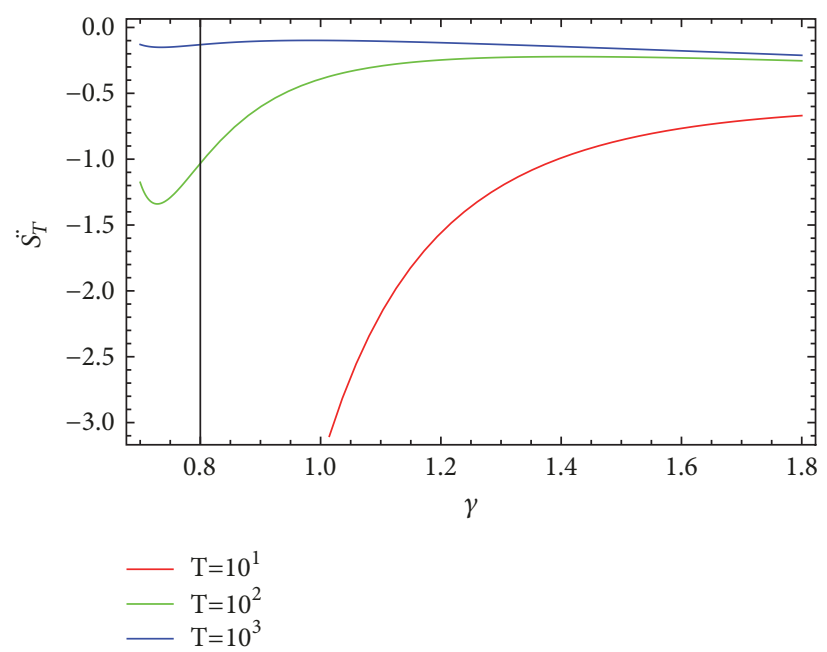

FIGURE 8: Plot of $\ddot{S_{T}}$ versus $\gamma$ for Renyi entropy when $\Gamma=$ constant.

The graphical behavior of $\overline{S_{T}}$ versus $\gamma$ for three values of $T$ is shown in Figure 8. It can be seen that $\dot{S}_{T}$ is negative for all values of $T$ satisfying the condition $d^{2} S_{T} / d t^{2}<0$ which exhibits the thermodynamical equilibrium.
4.2. $\Gamma=\Gamma(t)$. Secondly, we consider particle creation rate $\Gamma$ as a variable, i.e., $\Gamma=\Gamma(t)$.

Bekenstein Entropy. The differentiation of (35) leads to

$$
\begin{aligned}
\ddot{S_{T}} & =-\frac{3 \dot{H}\left(c^{2} / a^{6}+\gamma(1-\Gamma / 3 H)\left(-c^{2} / 2 a^{6}+3 H^{2}\right)\right)}{8 H^{4}}-2 \dot{H}\left(-\frac{c^{2}}{6 a^{6}}+H^{2}\right)^{(1-\gamma) / \gamma} \\
& \times\left(\frac{-\gamma\left(3 H^{2}-c^{2} / 2 a^{6}\right)(1-\Gamma / 3 H)+\gamma\left(3 H^{2}-c^{2} / 2 a^{6}\right)\left(c^{2} / a^{6}+\gamma\left(3 H^{2}-c^{2} / 2 a^{6}\right)(1-\Gamma / 3 H)\right) / 2 H^{2}}{H^{3} T_{0}}\right) \\
& +\left(\frac{-\gamma\left(3 H^{2}-c^{2} / 2 a^{6}\right)(1-\Gamma / 3 H)+\gamma\left(3 H^{2}-c^{2} / 2 a^{6}\right)\left(c^{2} / a^{6}+\gamma\left(3 H^{2}-c^{2} / 2 a^{6}\right)(1-\Gamma / 3 H)\right) / 2 H^{2}}{\gamma H^{2} T_{0}}\right) \times(1-\gamma) \\
& +\left(\frac{c^{2} \dot{a}}{a^{7}}+2 H \dot{H}\right)\left(H^{2}-\frac{c^{2}}{6 a^{6}}\right)^{-1+(1-\gamma) / \gamma}-\frac{6 c^{2} \dot{a}}{8 H^{3} a^{7}}+\frac{\left(3 c^{2} \dot{a} / a^{7}+6 H \dot{H}\right)}{8 H^{3}} \times \gamma\left(1-\frac{\Gamma}{3 H}\right) \\
& +\frac{\gamma\left(3 H^{2}-c^{2} / 2 a^{6}\right)\left(\Gamma \dot{H} / 3 H^{2}-\dot{\Gamma} / 3 H\right)}{8 H^{3}}+\frac{\left(H^{2}-c^{2} / 6 a^{6}\right)^{(1-\gamma) / \gamma}}{H^{2} T_{0}} \\
& \times\left(-\frac{\gamma \dot{H}\left(3 H^{2}-c^{2} / 2 a^{6}\right)\left(c^{2} / a^{6}+\left(3 H^{2}-c^{2} / 2 a^{6}\right)(1-\Gamma / 3 H)\right)}{H^{3}}-\gamma\left(1-\frac{\Gamma}{3 H}\right) \times\left(\frac{3 c^{2} \dot{a}}{a^{7}}+6 H \dot{H}\right)\right. \\
& +\frac{\gamma\left(c^{2} / a^{6}+\gamma\left(3 H^{2}-c^{2} / 2 a^{6}\right)(1-\Gamma / 3 H)\right)\left(3 c^{2} \dot{a} / a^{7}+6 H \dot{H}\right)}{2 H^{2}}-\gamma\left(3 H^{2}-\frac{c^{2}}{2 a^{6}}\right)\left(\frac{\Gamma \dot{H}}{3 H^{2}}-\frac{\dot{\Gamma}}{3 H}\right) \\
& \left.+\left(\frac{-6 c^{2} \dot{a} / a^{7}+\gamma(1-\Gamma / 3 H)\left(3 c^{2} \dot{a} / a^{7}+6 H \dot{H}\right)}{2 H^{2}}+\frac{\gamma\left(3 H^{2}-c^{2} / 2 a^{6}\right)\left(\Gamma \dot{H} / 3 H^{2}-\dot{\Gamma} / 3 H\right)}{2 H}\right)\left(3 H^{2}-\frac{c^{2}}{2 a^{6}}\right) \gamma\right) .
\end{aligned}
$$




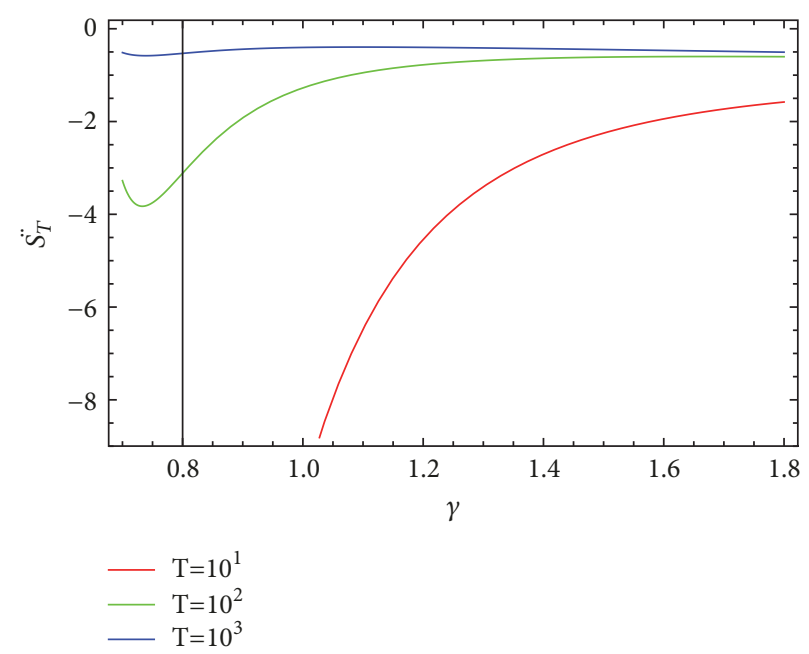

Figure 9: Plot of $\ddot{S}_{T}$ versus $\gamma$ for Bekenstein entropy when $\Gamma=\Gamma(t)$.

The plot of $\ddot{S_{T}}$ versus $\gamma$ for three values of $T$ is shown in Figure 9 by keeping the constant values same as for constant $\Gamma$. It is observed that the thermodynamical is obeying the condition $\dot{S}_{T}<0$ which leads to the validity of thermodynamical equilibrium.
Logarithmic Corrected Entropy. We discuss the stability analysis of thermal equilibrium in the presence of logarithmic corrected entropy by taking $\Gamma$ as variable for which (36) reduces to second-order differential equation as

$$
\begin{aligned}
\dot{S}_{T}^{*} & =-\frac{\dot{H}\left(1 / 4 H L_{p}^{2}+2 \alpha H-16 L_{p}^{2} \beta H^{3}\right)\left(c^{2} / a^{6}+\gamma(1-\Gamma / 3 H)\left(3 H^{2}-c^{2} / 2 a^{6}\right)\right)}{H^{3}} \\
& -\left(\frac{-\gamma(1-\Gamma / 3 H)\left(3 H^{2}-c^{2} / 2 a^{6}\right)+\gamma\left(3 H^{2}-c^{2} / 2 a^{6}\right)\left(c^{2} / a^{6}+\gamma(1-\Gamma / 3 H)\left(3 H^{2}-c^{2} / 2 a^{6}\right)\right) / 2 H^{2}}{H^{3} T_{0}}\right) \times 2 \dot{H}\left(H^{2}\right. \\
& \left.-\frac{c^{2}}{6 a^{6}}\right)^{(1-\gamma) / \gamma}+(1-\gamma)\left(\frac{c^{2} \dot{a}}{a^{7}}+2 H \dot{H}\right)\left(H^{2}-\frac{c^{2}}{6 a^{6}}\right)^{-1+(1-\gamma) / \gamma} \\
& +\left(\frac{-\gamma(1-\Gamma / 3 H)\left(3 H^{2}-c^{2} / 2 a^{6}\right)+\gamma\left(3 H^{2}-c^{2} / 2 a^{6}\right)\left(c^{2} / a^{6}+\gamma(1-\Gamma / 3 H)\left(3 H^{2}-c^{2} / 2 a^{6}\right)\right) / 2 H^{2}}{\gamma H^{2} T_{0}}\right) \\
& +\frac{\left(c^{2} / a^{6}+\gamma(1-\Gamma / 3 H)\left(3 H^{2}-c^{2} / 2 a^{6}\right)\right)\left(2 \alpha \dot{H}-\dot{H}^{6} / 4 L_{p}^{2} H^{2}-48 L_{p}^{2} \beta H^{2} \dot{H}\right)}{2 H^{2}}+\left(\frac{1}{4 H L_{p}^{2}}+2 \alpha H-16 L_{p}^{2} \beta H^{3}\right) \\
& +\left(\frac{-6 c^{2} \dot{a} / a^{7}+\gamma(1-\Gamma / 3 H)\left(3 c^{2} \dot{a} / a^{7}+6 H \dot{H}\right)}{2 H^{2}}+\frac{\gamma\left(3 H^{2}-c^{2} / 2 a^{6}\right)\left(\Gamma \dot{H} / 3 H^{2}-\dot{\Gamma} / 3 H\right)}{2 H^{2}}\right)+\frac{1}{H^{2} T_{0}}\left(H^{2}\right. \\
& \left.-\frac{c^{2}}{6 a^{6}}\right)^{(1-\gamma) / \gamma}\left(-\left(3 H^{2}-\frac{c^{2}}{2 a^{6}}\right) \times \frac{\gamma \dot{H}\left(c^{2} / a^{6}+\gamma(1-\Gamma / 3 H)\left(3 H^{2}-c^{2} / 2 a^{6}\right)\right)}{H^{3}}-\gamma\left(1-\frac{\Gamma}{3 H}\right)\left(\frac{3 c^{2} \dot{a}}{a^{7}}+6 H \dot{H}\right)\right. \\
& +\frac{\gamma\left(c^{2} / a^{6}+\gamma(1-\Gamma / 3 H)\left(3 H^{2}-c^{2} / 2 a^{6}\right)\right)\left(3 c^{2} \dot{a} / a^{7}+6 H \dot{H}\right)}{2 H^{2}}-\gamma\left(3 H^{2}-\frac{c^{2}}{2 a^{6}}\right) \times\left(\frac{\Gamma \dot{H}}{3 H^{2}}-\frac{\dot{\Gamma}}{3 H}\right) \\
& \left.+\frac{\gamma\left(3 H^{2}-c^{2} / 2 a^{6}\right)\left(-6 c^{2} \dot{a} / a^{7}+\gamma(1-\Gamma / 3 H)\left(3 c^{2} \dot{a} / a^{7}+6 H \dot{H}\right)\right)}{2 H^{2}\left(3 H^{2}-c^{2} / 2 a^{6}\right)^{2}\left(\Gamma \dot{H} / 3 H^{2}-\dot{\Gamma} / 3 H\right)} 2 H^{2}\right) .
\end{aligned}
$$




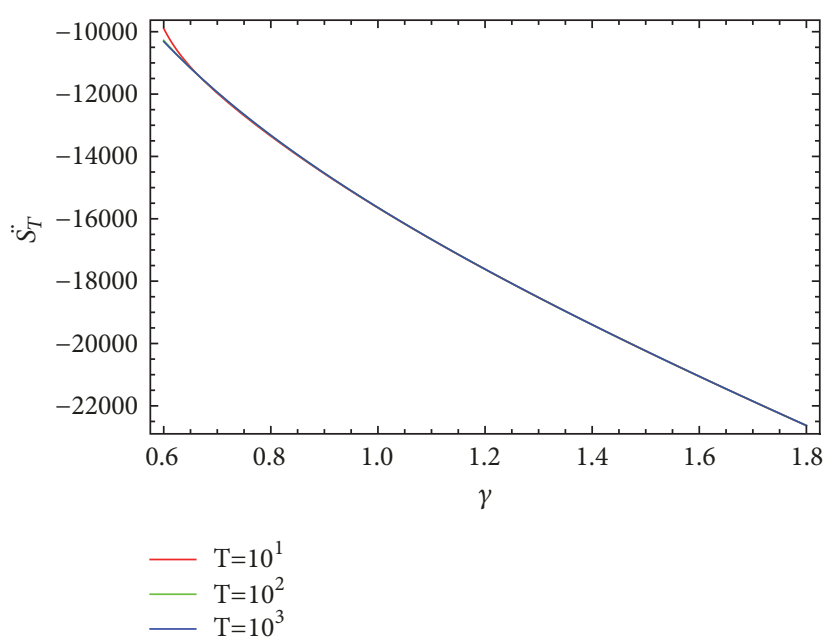

Figure 10: Plot of $\ddot{S_{T}}$ versus $\gamma$ when logarithmic corrected entropy $\Gamma=\Gamma(t)$.

The plot of $\dot{S}_{T}$ versus $\gamma$ is shown in Figure 10 for three values of $T$ by keeping the same values as in above case; we observe that thermal equilibrium condition $S_{T}<0$ is fulfilled which leads to the validity of thermodynamical equilibrium.

Power Law Correction. For variable $\Gamma$, the differentiation of (37) turns out to be

$$
\begin{aligned}
\ddot{S_{T}}= & -\frac{\left(1 / 4 H L_{p}^{2}-(2-\delta / 2)(1 / H)^{3-\delta} K_{\delta} / 4 L_{p}^{2}\right)\left(c^{2} / a^{6}+\gamma(1-\Gamma / 3 H)\left(3 H^{2}-c^{2} / 2 a^{6}\right)\right) \dot{H}}{H^{3}} \\
& -\left(\frac{-\gamma(1-\Gamma / 3 H)\left(3 H^{2}-c^{2} / 2 a^{6}\right)+\gamma\left(3 H^{2}-c^{2} / 2 a^{6}\right)\left(c^{2} / a^{6}+\gamma(1-\Gamma / 3 H)\left(3 H^{2}-c^{2} / 2 a^{6}\right)\right) / 2 H^{2}}{H^{3} T_{0}}\right) \times 2 \dot{H}\left(H^{2}\right. \\
& \left.-\frac{c^{2}}{6 a^{6}}\right)^{(1-\gamma) / \gamma}+(1-\gamma)\left(\frac{c^{2} \dot{a}}{a^{7}}+2 H \dot{H}\right)\left(H^{2}-\frac{c^{2}}{6 a^{6}}\right)^{-1+(1-\gamma) / \gamma} \\
& \times\left(\frac{-\gamma\left(3 H^{2}-c^{2} / 2 a^{6}\right)(1-\Gamma / 3 H)+\gamma\left(3 H^{2}-c^{2} / 2 a^{6}\right)\left(c^{2} / a^{6}+\gamma\left(3 H^{2}-c^{2} / 2 a^{6}\right)(1-\Gamma / 3 H)\right) / 2 H^{2}}{\gamma H^{2} T_{0}}\right) \\
& +\frac{\left(-\dot{H} / 4 H L_{p}^{2}+\dot{H}(3-\delta)(2-\delta / 2)(1 / H)^{4-\delta} K_{\delta} / 4 L_{p}^{2}\right)\left(c^{2} / a^{6}+\gamma\left(3 H^{2}-c^{2} / 2 a^{6}\right)(1-\Gamma / 3 H)\right)}{2 H^{2}}+\left(\frac{1}{4 H L_{p}^{2}}\right. \\
& \left.-\frac{(2-\delta / 2)(1 / H)^{3-\delta} K_{\delta}}{4 L_{p}^{2}}\right)\left(\frac{-6 c^{2} \dot{a} / a^{7}+\gamma(1-\Gamma / 3 H)\left(3 c^{2} \dot{a} / a^{7}+6 H \dot{H}\right)}{2 H^{2}}+\frac{\gamma\left(3 H^{2}-c^{2} / 2 a^{6}\right)\left(\Gamma \dot{H} / 3 H^{2}-\dot{\Gamma} / 3 H\right)}{2 H^{2}}\right) \\
& +\frac{\left(H^{2}-c^{2} / 6 a^{6}\right)^{(1-\gamma) / \gamma}}{H^{2} T_{0}}\left(-\left(\frac{3 c^{2} \dot{a}}{a^{7}}+6 H \dot{H}\right) \times \gamma\left(1-\frac{\Gamma}{3 H}\right)\right. \\
& +\frac{\gamma \dot{H}\left(3 H^{2}-c^{2} / 2 a^{6}\right)\left(c^{2} / a^{6}+\gamma\left(3 H^{2}-c^{2} / 2 a^{6}\right)(1-\Gamma / 3 H)\right)}{H^{3}} \\
+ & \frac{\gamma\left(3 c^{2} \dot{a} / a^{7}+6 H \dot{H}\right)\left(c^{2} / a^{6}+\gamma\left(3 H^{2}-c^{2} / 2 a^{6}\right)(1-\Gamma / 3 H)\right)}{2 H^{2}}-\gamma\left(3 H^{2}-\frac{c^{2}}{2 a^{6}}\right) \times\left(\frac{\Gamma \dot{H}}{3 H^{2}}-\frac{\dot{\Gamma}}{3 H}\right) \\
& \left.\left.\left.=\frac{\Gamma}{2}\right)\left(\frac{3 c^{2} \dot{a}}{a^{7}}+6 H \dot{H}\right)+\gamma\left(3 H^{2}-\frac{c^{2}}{2 a^{6}}\right)\left(\frac{\Gamma \dot{H}}{3 H^{2}}-\frac{\dot{\Gamma}}{3 H}\right)-\frac{6 c^{2} \dot{a}}{a^{7}}\right)\right) . \\
3 H & +\gamma\left(1-\frac{1}{6}\right)
\end{aligned}
$$




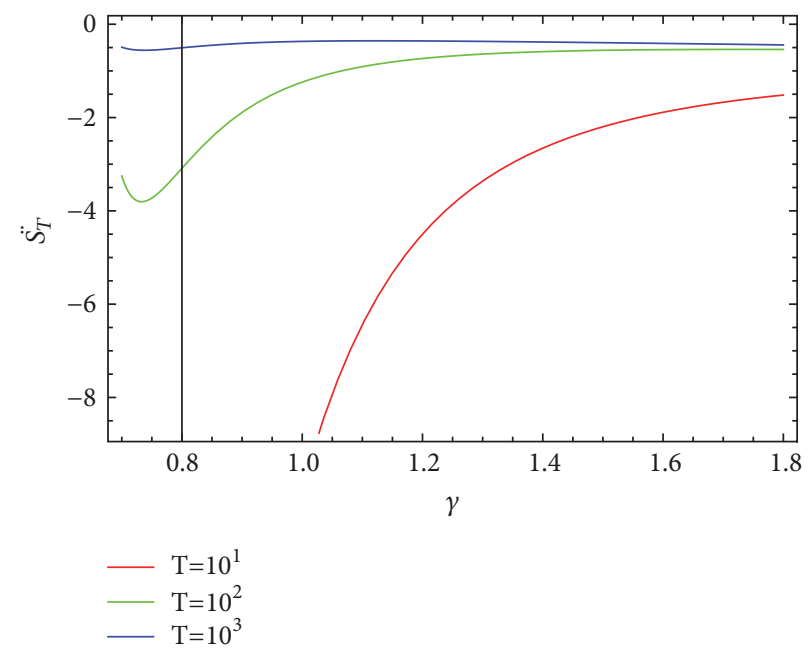

FIGURE 11: Plot of $\ddot{S_{T}}$ versus $\gamma$ for power law correction when $\Gamma=\Gamma(t)$.

The plot of $\ddot{S}_{T}$ versus $\gamma$ for three values of $T$ by keeping the same values is above mentioned (Figure 11); one can observe easily the validity of thermodynamical equilibrium for all values of $T$ with $S_{T}<0$.

Renyi Entropy. For variable Г, (38) gives

$$
\begin{aligned}
& \ddot{S_{T}}=-\frac{16 \dot{H}}{\left(\eta+8 H^{2}\right)^{2}}\left(\frac{c^{2}}{a^{6}}+\gamma\left(3 H^{2}-\frac{c^{2}}{2 a^{6}}\right)\left(1-\frac{\Gamma}{3 H}\right)\right)-\frac{\dot{H}}{H^{2}\left(\eta+8 H^{2}\right)} \times\left(\frac{c^{2}}{a^{6}}+\gamma\left(3 H^{2}-\frac{c^{2}}{2 a^{6}}\right)\left(1-\frac{\Gamma}{3 H}\right)\right) \\
& -\frac{2 \dot{H}\left(H^{2}-c^{2} / 6 a^{6}\right)^{(1-\gamma) / \gamma}}{H^{3} T_{0}}\left(\left(3 H^{2}-\frac{c^{2}}{2 a^{6}}\right) \times\left(1-\frac{\Gamma}{3 H}\right)(-\gamma)\right. \\
& \left.+\frac{\gamma\left(3 H^{2}-c^{2} / 2 a^{6}\right)\left(c^{2} / a^{6}+\gamma\left(3 H^{2}-c^{2} / 2 a^{6}\right)(1-\Gamma / 3 H)\right)}{2 H^{2}}\right) \\
& +\left(\frac{\gamma\left(3 H^{2}-c^{2} / 2 a^{6}\right)\left(c^{2} / a^{6}+\gamma\left(3 H^{2}-c^{2} / 2 a^{6}\right)(1-\Gamma / 3 H)\right)}{2 H^{2}}-\gamma\left(3 H^{2}-\frac{c^{2}}{2 a^{6}}\right) \times\left(1-\frac{\Gamma}{3 H}\right)\right) \\
& \cdot \frac{(1-\gamma)\left(c^{2} \dot{a} / a^{7}+2 H \dot{H}\right)\left(H^{2}-c^{2} / 6 a^{6}\right)^{-1+(1-\gamma) / \gamma}}{\gamma H^{2} T_{0}}+\frac{1}{H\left(\eta+8 H^{2}\right)} \times\left(\gamma\left(1-\frac{\Gamma}{3 H}\right)\left(\frac{3 c^{2} \dot{a}}{a^{7}}+6 H \dot{H}\right)\right. \\
& \left.+\gamma\left(3 H^{2}-\frac{c^{2}}{2 a^{6}}\right)\left(\frac{\Gamma \dot{H}}{3 H^{2}}-\frac{\dot{\Gamma}}{3 H}\right)-\frac{6 c^{2} \dot{a}}{a^{7}}\right) \\
& +\frac{\left(H^{2}-c^{2} / 6 a^{6}\right)^{(1-\gamma) / \gamma}}{H^{2} T_{0}}\left(-\frac{\gamma \dot{H}\left(3 H^{2}-c^{2} / 2 a^{6}\right)\left(c^{2} / a^{6}+\gamma\left(3 H^{2}-c^{2} / 2 a^{6}\right)(1-\Gamma / 3 H)\right)}{H^{3}}\right. \\
& +\frac{\gamma\left(3 c^{2} \dot{a} / a^{7}+6 H \dot{H}\right)\left(c^{2} / a^{6}+\gamma\left(3 H^{2}-c^{2} / 2 a^{6}\right)(1-\Gamma / 3 H)\right)}{2 H^{2}}-\gamma\left(\frac{3 c^{2} \dot{a}}{a^{7}}+6 H \dot{H}\right) \times\left(1-\frac{\Gamma}{3 H}\right) \\
& +\frac{\gamma\left(3 H^{2}-c^{2} / 2 a^{6}\right)}{2 H^{2}}\left(-\frac{6 c^{2} \dot{a}}{a^{7}}+\gamma\left(1-\frac{\Gamma}{3 H}\right)\left(\frac{3 c^{2} \dot{a}}{a^{7}}+6 H \dot{H}\right)+\gamma\left(3 H^{2}-\frac{c^{2}}{2 a^{6}}\right)\left(\frac{\Gamma \dot{H}}{3 H^{2}}-\frac{\dot{\Gamma}}{3 H}\right)\right) \\
& \left.-\gamma\left(3 H^{2}-\frac{c^{2}}{2 a^{6}}\right)\left(\frac{\Gamma \dot{H}}{3 H^{2}}-\frac{\dot{\Gamma}}{3 H}\right)\right) \text {. }
\end{aligned}
$$




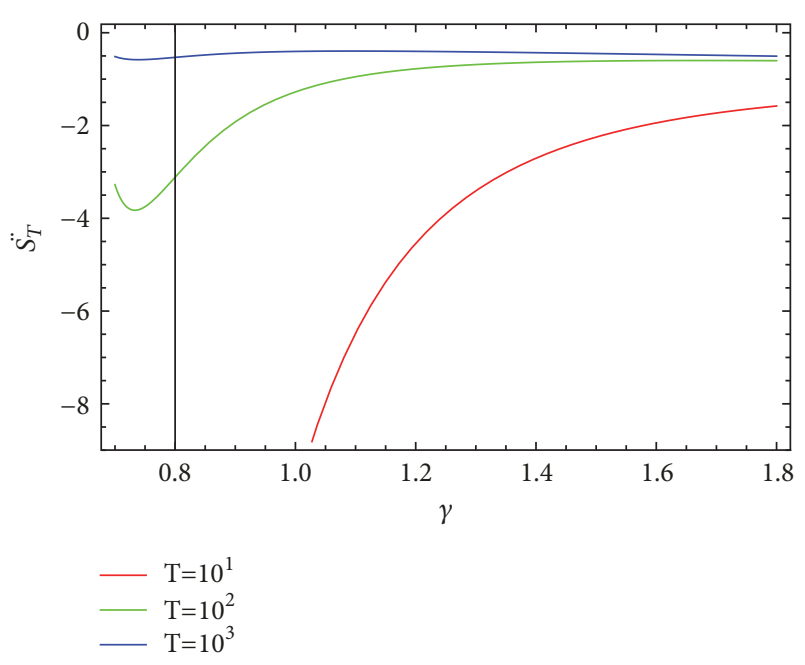

FIgURE 12: Plot of $\ddot{S_{T}}$ versus $\gamma$ for Renyi entropy when $\Gamma=\Gamma(t)$.

The graphical behavior of $\dot{S_{T}}$ versus $\gamma$ for three values of $T$ is shown in Figure 12 by keeping the constant values same as in above. One can see that $\vec{S}_{T}$ is negative for all values of $T$ satisfying the condition $d^{2} S_{T}<0$ which leads to the validity of thermodynamical equilibrium.

\section{Concluding Remarks}

In this work, we have investigated the validity of first law of thermodynamics, GSLT, and thermodynamical equilibrium for particle creation scenario in the presence of perfect fluid $\operatorname{EoS} p=(\gamma-1) \rho$ by assuming the different entropy corrections such as Bekenstein entropy, logarithmic corrected entropy and power law corrected entropy, and Renyi entropy in a newly proposed dynamical Chern-Simons modified gravity. We have summarized our results as follows:

(i) For Bekenstein entropy: we have analyzed that first law of thermodynamics is showing the validity for $\Gamma=3 H\left(\left(c^{2} / \gamma a^{6}\right)\left(3 H^{2}-c^{2} / 2 a^{6}\right)^{-1}\right)$. However, GSLT remains valid for all values of $T$ with $2 / 3 \leq \gamma \leq 2$. Further, we have analyzed the validity of thermodynamical equilibrium for constant and variable $\Gamma$. From Figures 5 and 9, we observe that thermodynamical equilibrium is satisfying the condition $d^{2} S_{T} / d t^{2}<0$ for all values of $T$ with $2 / 3 \leq \gamma \leq 2$.

(ii) For logarithmic corrected entropy:in the presence of logarithmic corrected entropy, it can be seen that the first law of thermodynamics is valid on the apparent horizon when $\Gamma=3 H\left(1-(1 / \gamma)\left(3 H^{2}-\right.\right.$ $\left.c^{2} / a^{6}\right)^{-1}\left(\gamma\left(3 H^{2}-c^{2} / 2 a^{6}\right) /\left(1 / L_{\mathrm{p}}^{2}+8 H^{2} \alpha-64 \beta H^{4} L_{\mathrm{p}}^{2}\right)-\right.$ $\left.\left.c^{2} / a^{6}\right)\right)$. We have also investigated the validity of GSLT on apparent horizon satisfying the condition $d S_{T} / d t \geq 0$ (Figure 2). The graphical behavior of $\ddot{S}_{T}$ versus $\gamma$ is shown in Figures 6 and 10. We observe the validity of thermodynamical equilibrium for all values of $T$, for all values of $\gamma$, for constant as well as variable $\Gamma$. (iii) Power law corrected entropy: for power law corrected entropy, we have investigated that first law of thermodynamics holds at apparent horizon for $\Gamma=3 H(1-$ $(1 / \gamma)\left(3 H^{2}-c^{2} / 2 a^{6}\right)^{-1}\left(\gamma\left(3 H^{2}-c^{2} / 2 a^{6}\right)\right)\left(1 / L_{\mathrm{p}}^{2}-(2-\right.$ $\left.\left.\delta / 2)\left(K_{\delta} / L_{\mathrm{p}}^{2}\right)(1 / H)^{2-\delta}\right)^{-1}-c^{2} / a^{6}\right)$. From Figure 3 we can analyze that the GSLT is valid for all values of $T$ with $2 / 3 \leq \gamma \leq 2$. Further, we have investigated the validity of thermodynamical equilibrium obeying the condition $\ddot{S_{T}}<0$ as shown in Figures 7 and 11 for all values of $T$ with $2 / 3 \leq \gamma \leq 2$ for both constant and variable $\Gamma$.

(iv) For Renyi entropy: in this entropy, we have observed that first law of thermodynamics holds when $\Gamma=$ $3 H\left(1-(1 / \gamma)\left(3 H^{2}-c^{2} / 2 a^{6}\right)^{-1}\left(\left(\gamma\left(\eta+8 H^{2}\right) / 8 H^{2}\right)\left(3 H^{2}-\right.\right.\right.$ $\left.\left.\left.c^{2} / 2 a^{6}\right)-c^{2} / a^{6}\right)\right)$. The graphical behavior of Figure 12 shows that all trajectories remain positive for all values of $T$ with $2 / 3 \leq \gamma \leq 2$ which leads to the validity of GSLT. Moreover, thermodynamical equilibrium condition is satisfied for all values of $T$ with all values of $\gamma$ for constant and variable $\Gamma$.

\section{Data Availability}

The data used to support the findings of this study are available from the corresponding author upon request.

\section{Conflicts of Interest}

The authors declare that they have no conflicts of interest.

\section{References}

[1] D. N. Spergel, R. Bean, and O. Doré, "Three-year Wilkinson Microwave Anisotropy Probe (WMAP) observations: implications for cosmology," The Astrophysical Journal Supplement Series, vol. 170, no. 2, p. 377, 2007.

[2] J. K. Adelman-McCarthy, M. A. Agüeros, and S. S. Allam, "The sixth data release of the sloan digital sky survey," The Astrophysical Journal Supplement Series, vol. 175, no. 2, p. 297, 2008.

[3] B. Ratra and P. J. E. Peebles, "Cosmological consequences of a rolling homogeneous scalar field," Physical Review D: Particles, Fields, Gravitation and Cosmology, vol. 37, no. 12, pp. 3406-3427, 1988.

[4] C. Armendáriz-Picón, T. Damour, and V. Mukhanov, " $k$ Inflation," Physics Letters B, vol. 458, no. 2-3, pp. 209-218, 1999.

[5] R. R. Caldwell, "A phantom menace? Cosmological consequences of a dark energy component with super-negative equation of state," Physics Letters B, vol. 545, p. 23, 2002.

[6] B. Feng, X. L. Wang, and X. M. Zhang, Physics Letters B, vol. 607, p. 35, 2005.

[7] T. Padmanabhan, Physical Review D, vol. 66, Article ID 021301, 2002.

[8] M. Li, "A model of holographic dark energy," Physics Letters B, vol. 603, p. 1, 2004.

[9] H. Wei, Classical and Quantum Gravity, vol. 29, Article ID 175008,2012

[10] A. Jawad and G. Abbas, "Interacting new agegraphic version of pilgrim dark energy," International Journal of Modern Physics D: 
Gravitation, Astrophysics, Cosmology, vol. 24, no. 8, 1550061, 20 pages, 2015.

[11] A. Jawad and A. Majeed, "Correspondence of pilgrim dark energy with scalar field models," Astrophysics and Space Science, vol. 356, no. 2, pp. 375-381, 2015.

[12] A. Jawad, "Analysis of generalized ghost pilgrim dark energy in non-flat FRW universe," The European Physical Journal C, vol. 74, article 3215, 2014.

[13] M. Sharif and Z. Yousaf, "Stability of regular energy density in Palatini $f(R)$ gravity, The European Physical Journal C, vol. 75, p. 58, 2015.

[14] J. Amorós, J. De Haro, and S. D. Odintsov, "Bouncing loop quantum cosmology from $F(T)$ gravity," Physical Review D: Particles, Fields, Gravitation and Cosmology, vol. 87, no. 10, Article ID 104037, 2013.

[15] G. Kofinas, G. Leon, and E. N. Saridakis, "Dynamical behavior in $\inf (T, T G)$ cosmology," Classical and Quantum Gravity, vol. 31, no. 17, Article ID 175011, 2014.

[16] S. Chattopadhyay, A. Jawad, D. Momeni, and R. Myrzakulov, "Pilgrim dark energy in $f\left(T, T_{G}\right)$ cosmology," Astrophysics and Space Science, vol. 35, p. 279, 2014.

[17] T. Harko, F. S. N. Lobo, G. Otalora, and E. N. Saridakis, " $F(T, T)$ gravity and cosmology," Journal of Cosmology and Astroparticle Physics, vol. 2014, no. 12, article no. 021, 2014.

[18] I. G. Salako, A. Jawad, and S. Chattopadhyay, "Holographic dark energy reconstruction in $f(T, \tau)$ gravity," Astrophysics and Space Science, vol. 358, no. 13, 2015.

[19] J. G. Silva and A. F. Santos, "Ricci dark energy in Chern-Simons modified gravity," The European Physical Journal C, vol. 73, article 2500, 2013.

[20] Y. S. Myung, "Comment on Ricci dark energy in Chern-Simons modified gravity," The European Physical Journal C, vol. 73, article 2515, 2013.

[21] A. Pasqua, R. da Rocha, and S. Chattopadhyay, "Holographic dark energy models and higher order generalizations in dynamical Chern-Simons modified gravity," The European Physical Journal C, vol. 75, article 44, 2015.

[22] A. Jawad, I. Salako, and A. Sohail, "Ghost dark energy models in specific modified gravity," The European Physical Journal Plus, vol. 131, 2016.

[23] M. Akbar and R. G. Cai, "Thermodynamic behavior of the Friedmann equation at the apparent horizon of the FRW universe," Physical Review D: Particles, Fields, Gravitation and Cosmology, vol. 75, Article ID 084003, 2007.

[24] R. G. Cai and L. M. Cao, "Unified first law and thermodynamics of apparent Horizon in FRW universe," Physical Review D, vol. 75, Article ID 064008, 2007.

[25] R. G. Cai and S. P. Kim, "First law of thermodynamics and friedmann equations of friedmann-robertson-walker universe," Journal of High Energy Physics, vol. 2005, no. 2, p. 50, 2005.

[26] A. V. Frolov and L. Kofman, "Inflation and de Sitter thermodynamics," Journal of Cosmology and Astroparticle Physics, vol. 2003, no. 05, pp. 009-009, 2003.

[27] U. K. Danielsson, "Transplanckian energy production and slow roll inflation,” Physical Review D, vol. 71, Article ID 023516, 2005.

[28] R. S. Bousso, "Cosmology and the S matrix," Physical Review D: Particles, Fields, Gravitation and Cosmology, vol. 71, no. 6, Article ID 064024, 2005.

[29] G. Calcagni, "de Sitter thermodynamics and the braneworld," Journal of High Energy Physics, vol. 2005, no. 09, pp. 060-060, 2005.
[30] B. Wang, E. Abdalla, and R. Su, "Relating Friedmann equation to Cardy formula in universes with cosmological constant," Physics Letters B, vol. 503, no. 3-4, pp. 394-398, 2001.

[31] B. Wang, E. Abdalla, and R. Su, "Friedmann equation and cardy formula correspondence in brane universes," Modern Physics Letters A, vol. 17, no. 01, pp. 23-29, 2002.

[32] R.-G. Cai and Y. S. Myung, "Holography in a radiationdominated universe with a positive cosmological constant," Physical Review D: Particles, Fields, Gravitation and Cosmology, vol. 67, no. 12, 124021, 10 pages, 2003.

[33] T. Jacobson, "Thermodynamics of spacetime: the Einstein equation of state," Physical Review Letters, vol. 75, p. 1260, 1995.

[34] C. Eling, R. Guedens, and T. Jacobson, "Nonequilibrium thermodynamics of spacetime," Physical Review Letters, vol. 96, no. 12, Article ID 121301, 2006.

[35] M. Akbar and R. G. Cai, "Friedmann equations of FRW universe in scalar-tensor gravity, $f(R)$ gravity and first law of thermodynamics," Physics Letters B, vol. 635, no. 1, pp. 7-10, 2006.

[36] M. Akbar and R.-G. Cai, "Thermodynamic behavior of field equations for $f(R)$ gravity," Physics Letters B, vol. 648, no. 2-3, pp. 243-248, 2007.

[37] T. Padmanabhan, "Classical and quantum thermodynamics of horizons in spherically symmetric spacetimes," Classical and Quantum Gravity, vol. 19, no. 21, pp. 5387-5408, 2002.

[38] T. Padmanabhan, "Gravity: a new holographic perspective," International Journal of Modern Physics D, vol. 15, p. 1659, 2006.

[39] A. Paranjape, S. Sarkar, and T. Padmanabhan, "Thermodynamic route to field equations in Lanczos-LOVelock gravity," Physical Review D: Particles, Fields, Gravitation and Cosmology, vol. 74, no. 10, Article ID 104015, 2006.

[40] D. Kothawala, S. Sarkar, and T. Padmanabhan, "Einstein's equations as a thermodynamic identity: the cases of stationary axisymmetric horizons and evolving spherically symmetric horizons," Physics Letters B, vol. 652, no. 5-6, pp. 338-342, 2007.

[41] T. Padmanabhan and A. Paranjape, "Entropy of null surfaces and dynamics of spacetime," Physical Review D: Particles, Fields, Gravitation and Cosmology, vol. 75, no. 6, Article ID 064004, 2007.

[42] A. Denner and J.-N. Lang, "The complex-mass scheme and unitarity in perturbative quantum field theory," The European Physical Journal C, vol. 75, no. 8, p. 206, 2015.

[43] A. Jawad, S. Rani, and M. Saleem, "Cosmological study of reconstructed $f(T)$ models," Astrophysics and Space Science, vol. 362, no. 4, article no. 63, 2017.

[44] A. Jawad, S. Rani, I. G. Salako, and F. Gulshan, "Pilgrim dark energy models in fractal universe," International Journal of Modern Physics D, vol. 26, Article ID 1750049, 2017.

[45] A. Jawad, S. Rani, and N. Azhar, "Entropy corrected holographic dark energy models in modified gravity," International Journal of Modern Physics D, vol. 26, Article ID 1750040, 2017.

[46] A. Jawad, S. Rani, I. G. Salako, and F. Gulshan, "Cosmological study in loop quantum cosmology through dark energy model," International Journal of Modern Physics D: Gravitation, Astrophysics, Cosmology, vol. 26, no. 2, 1750007, 16 pages, 2017.

[47] S. Rani, A. Jawad, I. G. Salako, and N. Azhar, "Non-flat pilgrim dark energy FRW models in modified gravity," Astrophysics and Space Science, vol. 361, p. 286, 2016.

[48] A. Jawad, S. Chattopadhyay, and S. Rani, "Viscous pilgrim $f(T)$ gravity models," Astrophysics and Space Science, vol. 361, p. 231, 2016. 
[49] K. Bamba and C.-Q. Geng, "Thermodynamics in $F(R)$ gravity with phantom crossing," Physics Letters B: Particle Physics, Nuclear Physics and Cosmology, vol. 679, no. 3, pp. 282-287, 2009.

[50] K. Bamba, C. Geng, S. Nojiri, and S. D. Odintsov, "Equivalence of the modified gravity equation to the Clausius relation," $E P L$ (Europhysics Letters), vol. 89, no. 5, p. 50003, 2010.

[51] K. Bamba and C.-Q. Geng, "Thermodynamics in $f(R)$ gravity in the Palatini formalism," Journal of Cosmology and Astroparticle Physics, vol. 2010, no. 6, article 014, 20 pages, 2010.

[52] K. Bamba and C. Q. Geng, "Thermodynamics of cosmological horizons in $f(T)$ gravity," Journal of Cosmology and Astroparticle Physics, vol. 2011, article 008, 2011.

[53] K. Bamba, R. Myrzakulov, S. Nojiri, and S. D. Odintsov, "Reconstruction of $f(T)$ gravity: rip cosmology, finite-time future singularities, and thermodynamics," Physical Review D: Particles, Fields, Gravitation and Cosmology, vol. 85, no. 10, Article ID 104036, 2012.

[54] K. Bamba, M. Jamil, D. Momeni, and R. Myrzakulov, "Generalized second law of thermodynamics in $f(T)$ gravity with entropy corrections," Astrophysics and Space Science, vol. 344, p. 259, 2013.

[55] K. Bamba, "Thermodynamic properties of modified gravity theories," International Journal of Geometric Methods in Modern Physics, vol. 13, no. 6, 1630007, 23 pages, 2016.

[56] S. Saha and A. Mondal, "Thermodynamic implications of the gravitationally induced particle creation scenario," The European Physical Journal C, vol. 77, no. 3, 2017.

[57] J. D. Bekenstein, "Black holes and entropy," Physical Review D: Particles, Fields, Gravitation and Cosmology, vol. 7, pp. 23332346, 1973.

[58] K. Karami, S. Ghaffari, and M. M. Soltanzadeh, "The generalized second law of gravitational thermodynamics on the apparent and event horizons in FRW cosmology," Classical and Quantum Gravity, vol. 27, Article ID 205021, 2010.

[59] M. R. Setare, "Interacting holographic dark energy model and generalized second law of thermodynamics in a non-flat universe," Journal of Cosmology and Astroparticle Physics, vol. 01, p. 23, 2007.

[60] A. Sheykhi, "Thermodynamics of interacting holographic dark energy with the apparent horizon as an IR cutoff," Classical and Quantum Gravity, vol. 27, no. 2, Article ID 025007, 2010.

[61] N. Mazumder and S. Chakraborty, "Validity of the generalized second law of thermodynamics of the universe bounded by the event horizon in holographic dark energy model," General Relativity and Gravitation, vol. 42, no. 4, pp. 813-820, 2010.

[62] M. Jamil, E. N. Saridakis, and M. R. Setare, "Thermodynamics of dark energy interacting with dark matter and radiation," Physical Review D: Particles, Fields, Gravitation and Cosmology, vol. 81, no. 2, Article ID 023007, 6 pages, 2010.

[63] R. G. Cai and S. P. Kim, "First law of thermodynamics and Friedmann equations of Friedmann-Robertson-Walker universe," Journal of High Energy Physics, vol. 2005, article 050, 2005.

[64] R. Banerjee and S. K. Modak, "Quantum tunneling, blackbody spectrum and non-logarithmic entropy correction for Lovelock black holes," Journal of High Energy Physics, vol. 2009, no. 11, pp. 073-073, 2009.

[65] H.-X. He, "Gauge symmetry and transverse symmetry transformations in gauge theories," Communications in Theoretical Physics, vol. 52, no. 2, pp. 292-294, 2009.
[66] A. Sheykhi and M. Jamil, "Power-Law entropy corrected holographic dark energy model," General Relativity and Gravitation, vol. 43, no. 10, pp. 2661-2672, 2011.

[67] E. Schrodinger, "The proper vibrations of the expanding universe," Physica A: Statistical Mechanics and its Applications, vol. 6, pp. 899-912, 1939.

[68] L. Parker, "Particle creation in expanding universes," Physical Review Letters, vol. 21, no. 8, pp. 562-564, 1968.

[69] L. Parker, "Quantized fields and particle creation in expanding universes. I," Physical Review A: Atomic, Molecular and Optical Physics, vol. 183, no. 5, pp. 1057-1068, 1969.

[70] N. D. Birrell and P. C. W. Davies, Quantum Fields in Curved Space, Cambridge University Press, Cambridge, UK, 1982.

[71] V. Mukhanov and S. Winitzki, Introduction to Quantum Effects in Gravity, Cambridge University Press, Cambridge, UK, 2007.

[72] L. E. Parker and D. J. Toms, Quantum Field Theory in Curved Spacetime: Quantized Field and Gravity, Cambridge Monographs on Mathematical Physics, Cambridge University Press, Cambridge, UK, 2009.

[73] I. Prigogine, "Thermodynamics and cosmology," General Relativity and Gravitation, vol. 21, p. 767, 1989.

[74] J. A. S. Lima, M. O. Calvao, and I. Waga, "Cosmology, Thermodynamics and Matter Creation," https://arxiv.org/abs/ 0708.3397 .

[75] J. Gariel and G. le Denmat, "Matter creation and bulk viscosity in early cosmology," Physics Letters A, vol. 200, no. 1, pp. 11-16, 1995.

[76] L. R. Abramo and J. A. Lima, "Inflationary models driven by adiabatic matter creation," Classical and Quantum Gravity, vol. 13, no. 11, pp. 2953-2964, 1996.

[77] G. Steigman, R. Santos, and J. Lima, "An accelerating cosmology without dark energy," Journal of Cosmology and Astroparticle Physics, vol. 2009, no. 06, pp. 033-033, 2009.

[78] S. Basilakos and J. A. S. Lima, "Constraints on cold dark matter accelerating cosmologies and cluster formation," Physical Review D: Particles, Fields, Gravitation and Cosmology, vol. 82, Article ID 023504, 2010.

[79] K. A. Meissner, "Black-hole entropy in loop quantum gravity," Classical and Quantum Gravity, vol. 21, no. 22, pp. 5245-5251, 2004.

[80] A. Ghosh and P. Mitra, "Ghosh and Mitra Reply," Physical Review Letters, Article ID 027502, 2004.

[81] A. Chatterjee and P. Majumdar, "Universal canonical black hole entropy," Physical Review Letters, vol. 92, no. 14, Article ID 141301, 2004.

[82] R. Banerjee and S. K. Modak, "Exact differential and corrected area law for stationary black holes in tunneling method," Journal of High Energy Physics, vol. 2009, no. 05, p. 63, 2009.

[83] S. K. Modak, "Corrected entropy of BTZ black hole in tunneling approach," Physics Letters B, vol. 671, no. 1, pp. 167-173, 2009.

[84] M. Jamil and M. U. Farooq, "Interacting holographic dark energy with logarithmic correction," Journal of Cosmology and Astroparticle Physics, vol. 2010, no. 3, p. 001, 2010.

[85] H. M. Sadjadi and M. Jamil, "Generalized second law of thermodynamics for FRW cosmology with logarithmic correction," EPL (Europhysics Letters), vol. 92, p. 69001, 2010.

[86] R. Banerjee and S. K. Modak, "Exact differential and corrected area law for stationary black holes in tunneling method," Journal of High Energy Physics, vol. 2009, no. 5, article 063, 32 pages, 2009. 
[87] R. Banerjee, S. Gangopadhyay, and S. K. Modak, "Voros product, noncommutative Schwarzschild black hole and corrected area law," Physics Letters B, vol. 686, no. 2-3, pp. 181-187, 2010.

[88] S. Das, S. Shankaranarayanan, and S. Sur, "Power-law corrections to entanglement entropy of horizons," Physical Review D: Particles, Fields, Gravitation and Cosmology, vol. 77, no. 6, 2008.

[89] B. Pourhassan, S. Upadhyay, and H. Farahani, "Thermodynamics of Higher Order Entropy Corrected SchwarzschildBeltrami-de Sitter Black Hole," General Physics, Article ID 093B06, 2017, https://arxiv.org/abs/1701.08650.

[90] T. S. Biro and V. G. Czinner, "A q-parameter bound for particle spectra based on black hole thermodynamics with Renyi entropy," Physics Letters. B. Particle Physics, Nuclear Physics and Cosmology, vol. 726, no. 4-5, pp. 861-865, 2013.

[91] V. Czinner and H. GIguchi, "Rényi entropy and the thermodynamic stability of black holes," Physics Letters B, vol. 752, p. 306, 2016.

[92] W. Zimdahl and D. Pavón, "Expanding universe with positive bulk viscous pressures?" Physical Review D: Particles, Fields, Gravitation and Cosmology, vol. 61, no. 10, 2000. 

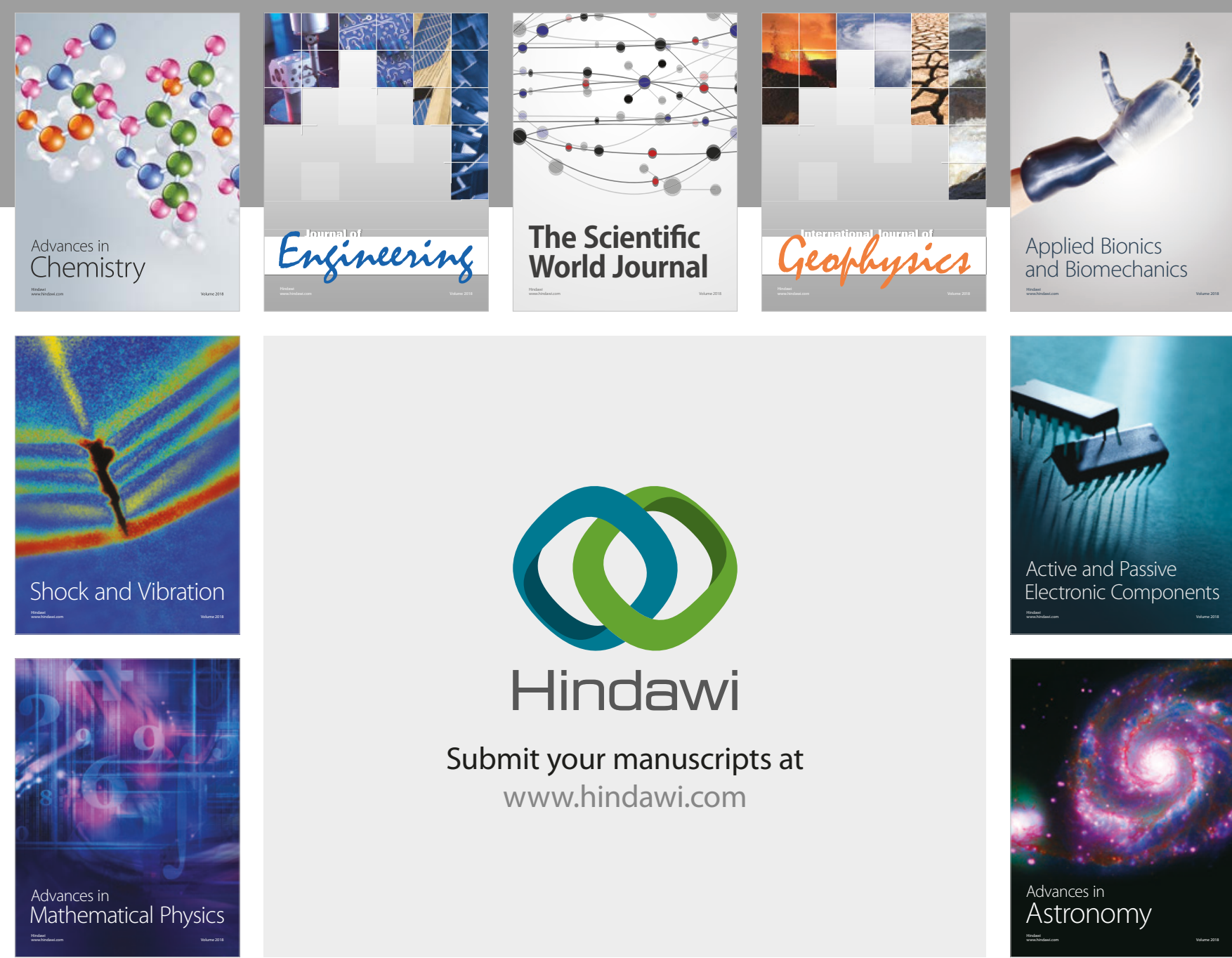

Submit your manuscripts at

www.hindawi.com

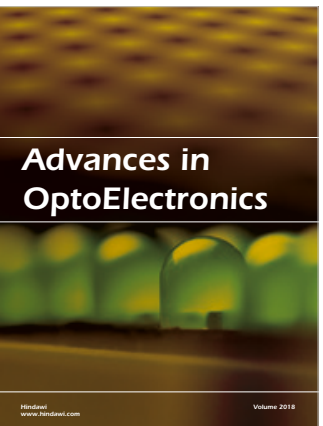

\section{Rotcting Machinery}
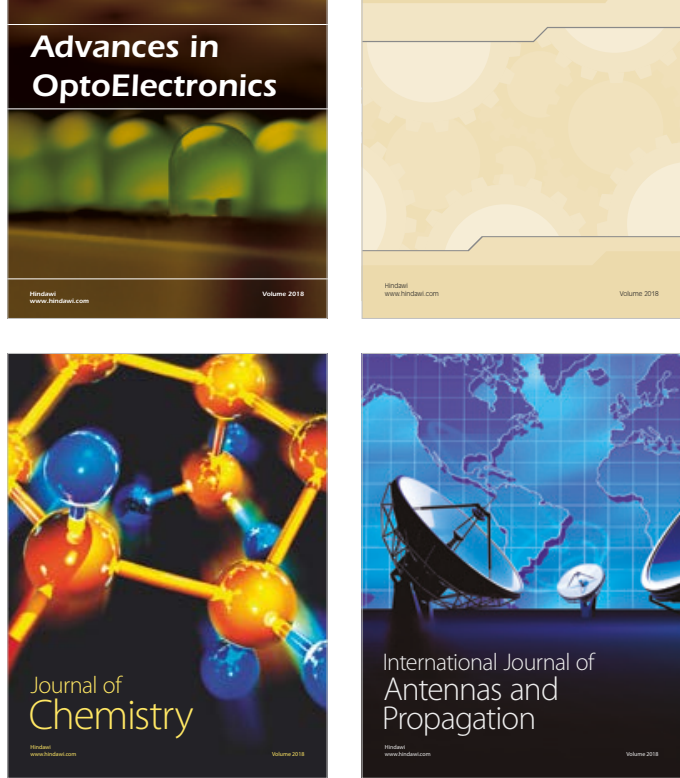

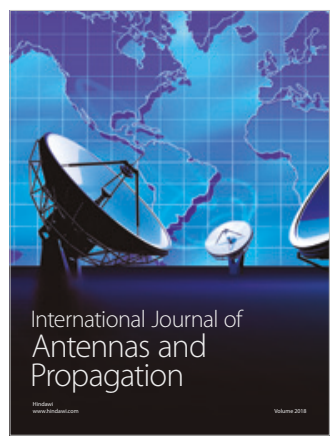

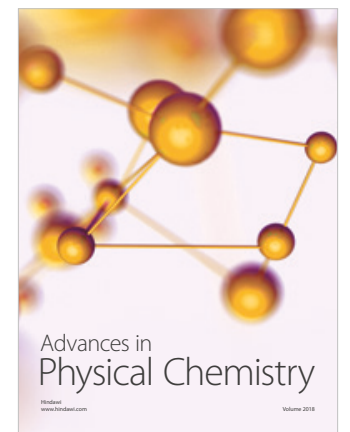

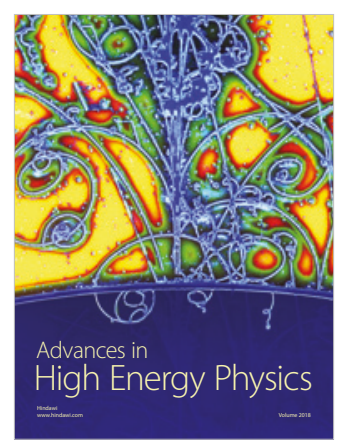

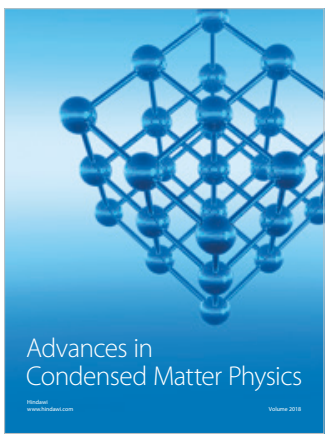

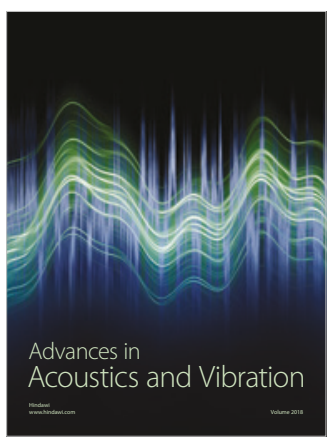

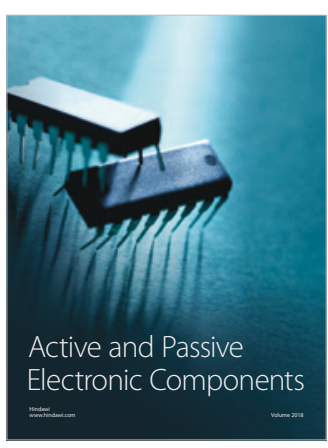
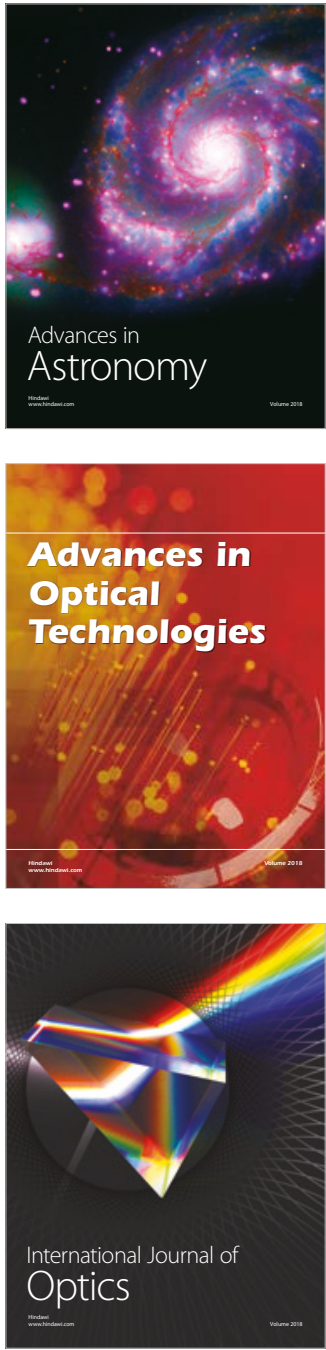